\title{
Cultural differences in affect intensity perception in the context of advertising
}

\author{
Marianna Pogosyan ${ }^{1}$ and Jan B. Engelmann ${ }^{2,3}$ * \\ ${ }^{1}$ Division of Public Administration, International Christian University, Tokyo, Japan \\ ${ }^{2}$ Laboratory for Social and Neural Systems Research, University of Zurich, Zurich, Switzerland \\ ${ }^{3}$ Department of Economics, University of Zurich, Zurich, Switzerland
}

\section{Edited by:}

Debi Roberson, University of Essex, UK

\section{Reviewed by:}

Ljubica Damjanovic, University of Chester, UK

Takeshi Hamamura, Chinese

Univerisity of Hong Kong, Hong Kong

*Correspondence:

Jan B. Engelmann, Department of Economics and Social Neural

Systems Laboratory, University of

Zurich, $\mathrm{CH}-8006$ Zurich, Switzerland

e-mail: jan.engelmann@econ.uzh.ch
Cultural differences in the perception of positive affect intensity within an advertising context were investigated among American, Japanese, and Russian participants. Participants were asked to rate the intensity of facial expressions of positive emotions, which displayed either subtle, low intensity, or salient, high intensity expressions of positive affect. In agreement with previous findings from cross-cultural psychological research, current results demonstrate both cross-cultural agreement and differences in the perception of positive affect intensity across the three cultures. Specifically, American participants perceived high arousal (HA) images as significantly less calm than participants from the other two cultures, while the Japanese participants perceived low arousal (LA) images as significantly more excited than participants from the other cultures. The underlying mechanisms of these cultural differences were further investigated through difference scores that probed for cultural differences in perception and categorization of positive emotions. Findings indicate that rating differences are due to (1) perceptual differences in the extent to which HA images were discriminated from LA images, and (2) categorization differences in the extent to which facial expressions were grouped into affect intensity categories. Specifically, American participants revealed significantly higher perceptual differentiation between arousal levels of facial expressions in high and intermediate intensity categories. Japanese participants, on the other hand, did not discriminate between high and low arousal affect categories to the same extent as did the American and Russian participants. These findings indicate the presence of cultural differences in underlying decoding mechanisms of facial expressions of positive affect intensity. Implications of these results for global advertising are discussed.

Keywords: cross-cultural differences, emotion perception, affect intensity, positive affect, Japan, USA, Russia

\section{INTRODUCTION}

Beginning from Darwinian assertions about the biological basis of emotions, the past century has witnessed a wealth of psychological research revealing universal tendencies in the mechanism of production and expression of emotions (e.g., Ekman et al., 1972; LeDoux, 1987; Buck, 1988; Grammer and Eibl-Eibesfeldt, 1993). Classical studies of emotion perception from the 1960 and 1970s known as the universality studies, have consistently documented universality of recognition of various emotional expressions including happiness, anger, disgust, fear, sadness, and surprise (e.g., Ekman and Friesen, 1969; Izard, 1971; Ekman et al., 1972). Subsequently, extensive research has revealed universality in the recognition of facial expressions among judges from a variety of cultural backgrounds, including American and Japanese judges (e.g., Matsumoto and Ekman, 1989; Matsumoto, 1990, 1992; Matsumoto et al., 1999) Hong Kong Chinese (e.g., Chan, 1985; Leung and Singh, 1998); Estonians, Germans, Greeks, Italians, Scottish, Sumatrans, Turks (e.g., Ekman et al., 1987), Indians (e.g., Mandal et al., 1986), and Australians (e.g., Toner and Gates, 1985; for a review, see Matsumoto, 2001). Furthermore, a number of studies have drawn attention to the possibility of adding contempt (e.g., Ekman and Heider, 1988) and embarrassment (e.g., Haidt and Keltner, 1999) to the list of universally recognizable facial expressions. Taken together, these findings provide evidence for the existence of universal processes in emotion recognition across cultures.

Recently, however, much attention has been given to the cultural contexts surrounding emotions and the possibility of a culturedependency of certain aspects of the affective mechanism (Russell, 1991). For instance, Matsumoto (1992) found that while American and Japanese judges showed cross-cultural convergence in the recognition of happiness and surprise, American judges were better at recognizing anger, disgust, fear, and sadness than their Japanese counterparts (see also Izard, 1971; Ekman et al., 1987; Matsumoto and Ekman, 1989). Furthermore, cross-cultural differences have also been reported in intensity ratings of emotions and their facial expressions (e.g., Ekman et al., 1987; Matsumoto, 1990; Biehl et al., 1997; Yrizarry et al., 1998; Matsumoto et al., 2002). Specifically, Japanese judges have repeatedly been found to rate facial expressions of various emotions, including happiness, sadness, and surprise, less intensely compared to American judges (e.g., Ekman et al., 1987; Matsumoto and Ekman, 1989; Biehl et al., 
1997). Interestingly, these differences in intensity ratings persisted regardless of (a) culture or gender of the face judged, (b) emotion judged, and (c) whether intensity was judged on a particular emotion or an affect-neutral intensity scale (Matsumoto and Ekman, 1989).

Such cross-cultural variability of emotion perception has been attributed to culturally imposed emotion display rules (Ekman and Friesen, 1969; Buck, 1988; Matsumoto et al., 2002). Display rules are learned naturally during childhood through socialization and subsequently determine the appropriateness of emotional behavior in various settings within given cultural contexts (Safdar et al., 2009). Prior research has demonstrated that the expression of feelings is encouraged in Western cultures, which typically score high on individualistic values, while in Asian cultures that are on average more collectivistic, the control of emotion is encouraged to maintain group harmony (e.g., Markus and Kitayama, 1991; Heine et al., 1999). Accordingly, the Japanese are tuned to avoid expressing intense emotions, since such emotions may pose a threat to group harmony and conformity (Matsumoto, 1992). Similarly, Russian nationals have been shown to engage in greater levels of affect expression control relative to South Koreans, Japanese, and Americans (Matsumoto et al., 1998). This result is qualified by high levels of affect control toward in-group members, such as family, friends, and colleagues, and relatively low levels of control toward out-group members (Matsumoto et al., 1998), underlining Russia's generally collectivistic attitudes. On the other hand, in individualistic cultural contexts, such as that of the United States, the suppression of emotions is viewed negatively (e.g., Markus and Kitayama, 1991; Heine et al., 1999) and the expression of not only positive but also negative emotions is encouraged (Matsumoto, 1992). Recent results have confirmed this initial finding by demonstrating significantly lower levels of self-reported affect control among Americans, compared to Russian and Japanese nationals (Matsumoto et al., 1998, 2005) and higher levels of expressivity of affect (Matsumoto et al., 2005).

Cultural display rules can have a significant impact on affect perception, as demonstrated in two recent studies (Yuki et al., 2007; Damjanovic et al., 2010). In their study, Yuki et al. (2007) tested the hypothesis that, when interpreting emotions of others, people from cultures with display rules requiring high levels of affect control focus more on areas of the face that are relatively difficult to control and thereby carry more information about true emotional states, such as the eyes, while people from cultures with less strict display rules focus on the mouth. Using cartoons and actual faces in which eye and mouth regions were independently manipulated, the authors showed that for conflicting stimuli (e.g., sad eyes together with happy mouth) Japanese participants gave more weight to the eyes, while American participants gave more weight to the mouth. These results provide strong evidence for the notion that cultural display rules can have a significant impact on the types of facial cues employed to interpret emotion. Further support for this notion is provided by more recent results from Damjanovic et al. (2010). Employing an affective visual search task with same and other race targets, the authors showed a facilitatory effect of the previously established happiness advantage (e.g., Calvo and Nummenmaa, 2008) when the visual search display consisted of other race targets. This result is in agreement with two prior findings on affective visual search, namely (1) that other race targets are processed featurally (Levin, 2000) while same race faces are processed holistically (e.g., Michel et al., 2006), and (2) that featural processing strategies facilitate the detection of happy facial expressions (Calvo and Nummenmaa, 2008). Therefore, results from Damjanovic et al. (2010) are consistent with the view that detection of discordant happy targets is improved in the context of other race faces via emphasizing feature-based search strategies. While this effect was obtained for English participants under various conditions, for Japanese participants such facilitatory effects only occurred during task instructions that specifically encouraged perceptual based strategies. The authors argue that Japanese participants did not show a happy face advantage for other race targets under conditions that emphasize emotion detection, while English participants did, due to differences in cultural display rules. Specifically, a perceptually salient smile that is incongruent with Japanese display rules can lead to interference during the detection of affect, as it might not match the prototype emotion during visual search (e.g., Roberson et al., 2007). Taken together, these results support the notion that cultural display rules can have significant modulatory effects on affect perception by modifying featural salience (Yuki et al., 2007), as well as perceptual processing strategies (Damjanovic et al., 2010).

To date, relatively little research has been conducted on cultural differences in affect intensity perception (see however Matsumoto and Ekman, 1989; Matsumoto, 1993; Yrizarry et al., 1998; Matsumoto et al., 1999, 2002). The present study aimed at extending existing findings on cross-cultural differences in affect intensity perception by addressing various shortcomings of previous research. Specifically, we investigated perception of emotion intensity across cultures within a particular context. The significance of context in the study of emotion has been emphasized in a series of recent studies that have revealed the modulatory effects that social and situational context has on emotion perception across different cultures (e.g., Masuda et al., 2008). The differences in contextual influences on the interpretations of emotions among Asians and Americans (e.g., Masuda et al., 2008; Ko et al., 2011) have been attributed to "analytic" versus "holistic" patterns of attention distribution among Western Europeans and East Asians (Nisbett et al., 2001). As such, Japanese and Korean participants have been shown to be influenced to a larger degree by contextual information when interpreting emotions compared to their North American peers.

Previous studies investigating affect intensity perception have asked participants to rate affect intensity within a contextual vacuum. In a typical setting of affect intensity rating studies, full-face, high intensity prototypical expressions are presented as stimuli, which participants are asked to judge categorically, as well as on intensity levels (Matsumoto, 2001; Matsumoto et al., 2002). Two important shortcomings of this standard approach come to mind: (1) the lack of interpretative context removes such experimental settings from real life situations, and (2) in everyday contexts, full-face and high intensity prototypical expressions such as those employed in prior experiments rarely occur and are thus more likely to reflect a controlled and weighted version of the portrayed emotions (e.g., Matsumoto et al., 2002). These shortcomings were addressed in the present study by employing stimuli with both high and low intensity expressions of affect and embedding the 
stimuli used in the present experiment in a situational context of advertising, thereby increasing the ecological validity of the study.

Advertising was chosen as the contextual platform for the investigation of affect intensity perception across cultures for a number of reasons. Firstly, print advertisements presented a setting that is ubiquitous within all three cultures included in the current study, thus providing a more naturalistic setting for judging facial expressions. Secondly, the ads featured professional models with significant practice in controlling facial expressions, which made the portrayed emotions seem more genuine and disposed of the artificiality that may occur with unpracticed posers. Finally, since standardization of print advertising has become an increasingly attractive option in international marketing due to financial and executive advantages, an in-depth examination of consumers' interpretation of affective messages used in ads could lend insight into the cross-cultural effectiveness of standardized ad campaigns.

The present study furthermore offers a different approach from past research of emotion perception by investigating cross-cultural differences in the perception of positive affect only, instead of examining the perception of multiple emotions of varying valence. By doing so, a more focused investigation of facial expressions of positive affect allowed us to include a wider range of emotion adjectives as response alternatives (Yrizarry et al., 1998). This approach yielded finer grained intensity ratings compared to previous studies that use simple categorical judgments, while at the same time avoiding the pitfalls of open-ended response formats, such as potential difficulties in classifying responses (Russell et al., 1993). Secondly, specific intensity ratings for different arousal categories of positive affect were collected simultaneously, which allowed us to measure perception of multiple arousal dimensions in response to viewing each image. Thirdly, the current study explored the perception of varying levels of positive affect among three cultures: the United States, Russia, and Japan, thus including participants from two well-studied cultures for comparison with previous research (United States and Japan) and participants from one relatively underrepresented culture in crosscultural research (Russia) to extend previous findings. Finally, only female participants were included in the current study, thus allowing us to use advertisements from the beauty industry, which provided an optimal pool of stimuli for the study of positive affect perception.

\section{OVERVIEW OF THE STUDY}

American, Japanese, and Russian female participants completed an online questionnaire in which they viewed beauty advertisements depicting models with positive emotional expressions. Each model was presented in two conditions, portraying either high arousal (HA; i.e., highly salient positive facial expressions) or low arousal (LA; i.e., more subtle positive facial expressions) positive emotions. Images from real advertisements were employed in order to control for attractiveness, familiarity, and authenticity of the portrayed affective expressions. Participants were asked to rate each advertisement on a novel rating scale based on the Affect Valuation Index (Tsai et al., 2006).

The main goal of the current investigation was to test the hypothesis that cultural differences exist in the perception of positive affect intensity and to explore the underlying mechanisms leading to those cultural differences. Due to previously reported differences in cultural display rules (e.g., Matsumoto and Ekman, 1989; Matsumoto et al., 1998, 2005) and their suggested influence on perception (e.g., Yuki et al., 2007; Damjanovic et al., 2010), we expected cross-cultural differences in intensity ratings for highly salient facial expressions, as well as subtle, LA facial expressions of positive affect. Specifically, due to higher levels of expressivity endorsement for happiness compared to other cultures (Matsumoto et al., 2005, 2008), we expected Americans to differ from Japanese and Russians in their ratings of high and low arousal positive (LAP) affect. Japanese and Russian nationals, on the other hand, have been shown to exert significantly higher levels of control over their displays of happiness compared to Americans (Matsumoto et al., 1998, 2005; Matsumoto, 2001) and additionally, are located in close proximity on the Individualism-Collectivism continuum (Matsumoto et al., 2008). Given these similarities, we expected participants from these cultures to have comparable percepts of LA affect. However, Japanese and Russian nationals have also been shown to differ in the way that affect is controlled, with Russians qualifying their emotions to a greater degree than Japanese and Americans, while Japanese deamplifying their emotions more than other cultures (Matsumoto et al., 2005). Such differences in display rules lead to disparate modifications of HA affect displays, with reductions of HA expressions in the case of deamplification and blended emotion in the case of qualification. We therefore expected differences in affect intensity perception for HA emotions between Japanese and Russian participants.

\section{MATERIALS AND METHODS PARTICIPANTS}

A total of 61 female participants were included in the current study: 22 participants from the United States, 18 from Japan, and 21 from Russia. Participants reported their age, nationality, current country of residence, years of education, and employment status. Demographic data for age and education (see Table 1) were entered into separate one-way Analyses of Variance (ANOVA) to test for cultural differences. Participants were well matched in the number of years of education $[F(2,58)=1.198, p=0.309]$, and age $[F(2,58)=1.091, p=0.343]$.

\section{STIMULI}

Eleven pairs of fictitious beauty advertisements were created that featured the same model portraying high arousal positive (HAP) and LAP emotions (see Figure 1). To increase the applicability of the findings, ads were selected from existing real print advertisements for cosmetics featured in global marketing campaigns between the years 2006 and 2008 and were slightly manipulated to create novel advertisements (e.g., Thorson, 1990). From a larger

Table 1 | Sample characteristics of participants from America, Russia, and Japan.

\begin{tabular}{llll}
\hline Variable & Americans & Russians & Japanese \\
\hline$N$ & 22 & 21 & 18 \\
Mean age (years) & 26.5 & 25.14 & 24.44 \\
Mean education (years) & 16.68 & 15.95 & 16.11
\end{tabular}



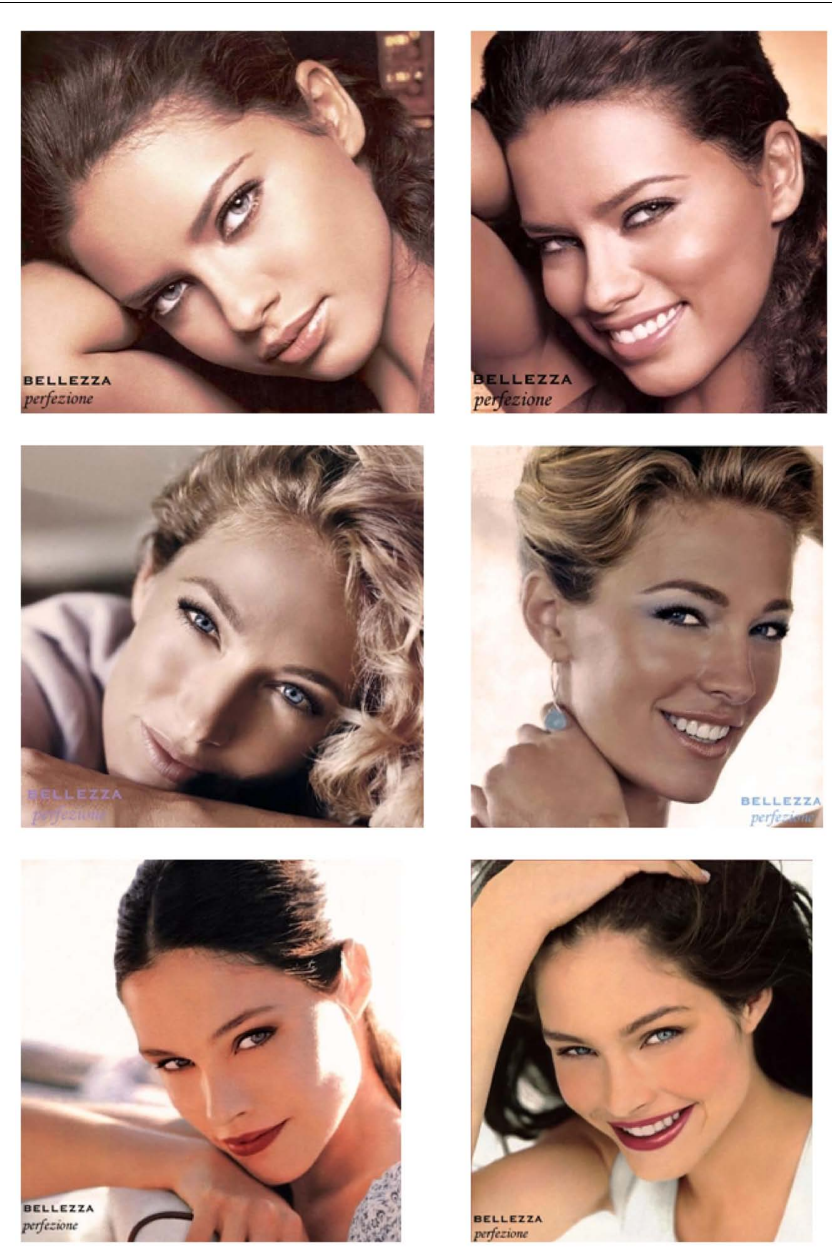

FIGURE 1 | Representative advertisements employed in the current investigation showing model pairs in LA (left side) and HA (right side) conditions.

set of available advertisements, a subset of advertisements was selected by one of the authors to create image pairs depicting the same model expressing a relatively higher arousal positive emotion in one image and a relatively lower arousal positive emotion in the other. For each model pair, we required the image classified as HA to depict (1) raised cheeks, and (2) raised lip corners relative to the image classified as LA. According to the Facial Action Coding System (FACS, Ekman and Friesen, 1978), these requirements are reflective of greater intensity in cheek raiser (Action Unit 6) and lip corner puller (Action Unit 12), respectively, which are prototypical action units used for identifying positive affect (see for instance Scherer and Ellgring, 2007; Krumhuber and Scherer, 2011). Finally, we required LA images to depict a closed-mouth smile and HA images to depict an open-mouth smile, which requires an increased jaw drop (Action Unit 26) in the HA image. Subsequently, emotion intensity categorization was independently confirmed by the co-author for all model pairs and only model pairs with the greatest level of agreement were included in the current experiment. A post hoc validation analysis that confirms significantly greater average
HA (HAP) ratings for HA facial expressions compared to LA facial expression tested individually for each model pair independent of culture is reported in Section "Appendix" (Table A1 in Appendix).

While the images of the ads remained the same as in the original ads, in order to control for familiarity and potential brand preference biases, all original verbal information was erased and replaced with the fictitious name of a new brand Bellezza and the catch phrase "Perfezione."

\section{INSTRUMENTS}

At the beginning of the study, respondents were informed that they are participating in a marketing research study about the perceptions of advertisement and that their responses will be used to select one of the ads in a marketing campaign for a novel product. Participants were deliberately kept unaware of the current investigation's focus on affect perception in order to control for demand effects. Participants were then asked to indicate how much they thought the model featured in each ad was portraying each one of the nine emotions provided in the questionnaire. These emotions varied in their intensity levels of positive affect and included three HAP emotions (excited, elated, enthusiastic), three Positive (POS) emotions (happy, content, satisfied), and three LAP emotions (calm, relaxed, peaceful). Participants were asked to rate the intensity of the emotions portrayed on a 5-point Likert scale, where one represented "this emotion is not being portrayed at all" and five represented "this emotion is being portrayed very strongly." Following methods from Tsai et al. (2006), ratings for emotions within the same intensity category (i.e., HAP, POS, LAP) were averaged and included in statistical analyses as the factor intensity category. The original questionnaire and instructions were created in English and translated and back-translated into Russian and Japanese by two native speakers from each country. At the end of the study, participants were informed of the goal of the study and given the chance to ask questions.

\section{PROCEDURE}

Advertisements and questionnaires were posted on designated websites using commercially available online survey websites (www.surveymonkey.com for English questionnaires and www.questionpro.com for Japanese and Russian questionnaires). The links to the surveys were distributed at different universities in the United States (Brown University), Japan (International Christian University), and Russia (Moscow State Institute of International Relations). While questions and advertisements were presented in the same order in each culture, the order of emotion adjectives was randomized for each participant.

\section{RESULTS}

\section{OUTLINE OF STATISTICAL ANALYSES}

In all analyses reported below (mean ratings, perceptual difference scores, categorization difference scores) and in Section "Appendix" (re-analyses based on ipsatized scores), a multivariate approach was employed since the Sphericity assumption required by repeated-measures univariate analyses was not met by the data, as indicated by significant results yielded by Mauchly's Test of Sphericity [intensity category: $p<0.05$; arousal 
level $\times$ intensity category: $p<0.001]$. In such cases, the multivariate approach is preferable to univariate analyses with correction, as it does not rely as heavily on assumptions (e.g., Maxwell and Delaney, 2000). Test statistics reported below, therefore, represent exact statistics obtained via Wilk's lambda and reported effect size reflects partial $\eta^{2}$. Marginally non-significant effects $(0.05<p \leq 0.1)$ are reported, and $p$-values for post hoc tests conducted for between-subjects factors reflect results from Fisher's LSD test.

The following analysis sequence was employed to investigate differential effects of culture on affect intensity perception and categorization: (1) a mixed omnibus MANOVA was conducted to probe for higher-order interactions with the factor culture, indicating that cultural background differentially impacted affect intensity perception and categorization, and (2) follow-up tests were conducted to further explore the nature of significant interactions. Given our hypothesis that culture differentially affects emotion intensity perception, a particular emphasis was placed on understanding the highest-order interaction, which qualifies lower-order interactions and main effects. The focus of follow-up tests was on the effects of culture on mean ratings or mean rating differences across relevant factor combinations. To this end, we first tested general effects of culture via a between-subjects MANOVA with culture as predictor variable and multiple dependent variables reflective of all factor combinations for a given analysis. Furthermore, provided that all previous multivariate tests were significant, univariate tests of simple effects were conducted to isolate mean ratings or rating differences that were significantly affected by culture, followed by post hoc tests (Fischer's LSD) to identify the exact cultural differences for significant simple effects.

In order to control for potential cultural differences in rating styles and response set bias (e.g., Hofstede, 1980; Chen et al., 1995), all data were standardized and reanalyzed (see Appendix for details). Specifically, to control for two types of bias that could systematically affect emotion ratings across cultures, namely (a) an extreme response style that is characterized by a tendency to use the endpoints of a scale, and (b) acquiescence response bias that is characterized by a shift to either end of a scale, we employed ipsatization as our method of standardizing raw scores. Results from additional analyses of the data support the trends reported in the analyses of the raw data and are reported in Section "Appendix" (see Tables A2-A11 and Figures A1-A3 in Appendix).

\section{CULTURAL DIFFERENCES IN EMOTION PERCEPTION}

To investigate cultural differences in the perception of positive affect intensity, mean ratings in each emotion intensity category were entered into a three-way mixed Multivariate Analysis of Variance (MANOVA), with arousal level (HA vs. LA facial expression) and intensity category (HAP, POS, LAP) as withinsubjects factors as well as culture (American, Russian, Japanese) as between-subjects factor. A significant main effect of intensity category was obtained $\left[F(2,57)=74.747, p<0.001, \eta^{2}=0.725\right]$, with highest ratings in the POS (3.291) intensity category compared to HAP (2.775) and LAP (2.887). There was also a significant main effect of arousal level $[F(1,58)=107.337, p<0.001$, $\left.\eta^{2}=0.649\right]$, with larger mean ratings for advertisements depicting HA (3.279) compared to LA (2.691) facial expressions. The main effect of culture did not reach significance $[F(2,58)=1.646$, $\left.p=0.202, \eta^{2}=0.054\right]$, but interacted significantly with the factors (1) arousal level $\left[F(2,58)=5.306, p<0.01, \eta^{2}=0.155\right]$, and (2) intensity category $\left[F(4,116)=3.614, p<0.01, \eta^{2}=0.113\right]$. Follow-up tests of the culture by arousal level interaction indicated that, for LA faces, American participants showed significantly lower mean ratings across all rating categories than both Japanese (Fisher's LSD mean difference was significant at $p<0.01$ ) and Russian participants $(p<0.05)$. The culture by intensity category interaction was driven by cultural differences in the HAP domain, such that American participants showed significantly lower mean ratings across both arousal levels than Japanese $[p<0.05]$ and Russian $[p<0.01]$ participants. A further two-way interaction was obtained between arousal level and intensity category $\left[F(2,57)=67.529, p<0.001, \eta^{2}=0.703\right]$. Post hoc pairwise comparisons investigating differences between intensity categories for a given arousal level, as well as comparisons within a given intensity category, but across arousal levels, were significant at $p<0.001$, except when comparing mean LAP ratings in response to high vs. LA faces, for which a marginally non-significant $p$ value $[p=0.091]$ was obtained. Finally, in agreement with the hypothesis of the present study, a significant three-way interaction was found between the factors arousal level, intensity category, and culture $\left[F(4,114)=6.641, p<0.001, \eta^{2}=0.189\right.$; Figure 2] . Because lower-order interactions are best described in terms of significant higher-order interactions, the nature of the three-way interactions was investigated in detail in two ways: (a) by means of tests of simple effects in combination with post hoc pairwise comparisons testing the effects of culture on ratings within each intensity category as a function of arousal level, and (b) by means of difference scores comparing (1) differences between mean ratings for each arousal level within each intensity category, and (2) differences between mean ratings for each emotion intensity category within each arousal level. Difference scores not only summarize the three-way interaction between culture, arousal level, and intensity category, but also allow for investigating the effects of CULTURE on emotion intensity perception and emotion intensity categorization.

\section{Tests of simple effects}

Because the focus of the current study is the effect of culture on intensity ratings of facial expressions of positive affect, we examined the three-way interaction between arousal level, intensity category, and culture more closely by investigating how culture affected mean ratings. We first probed for general effects of culture on ratings within each intensity category as a function of arousal level. To this end, a between-subjects MANOVA was performed with culture as predictor variable and ratings across intensity category and arousal level as multiple dependent variables (mean ratings within the following factor combinations between intensity category and arousal level were included as dependent variables: mean ratings in the HAP domain for LA facial expressions = HAP_LA, as well as POS_LA, LAP_LA, HAP_HA, POS_HA, LAP_HA). A significant effect of culture $\left[F(12,106)=5.396, p<0.001, \eta^{2}=0.379\right]$ was obtained, 


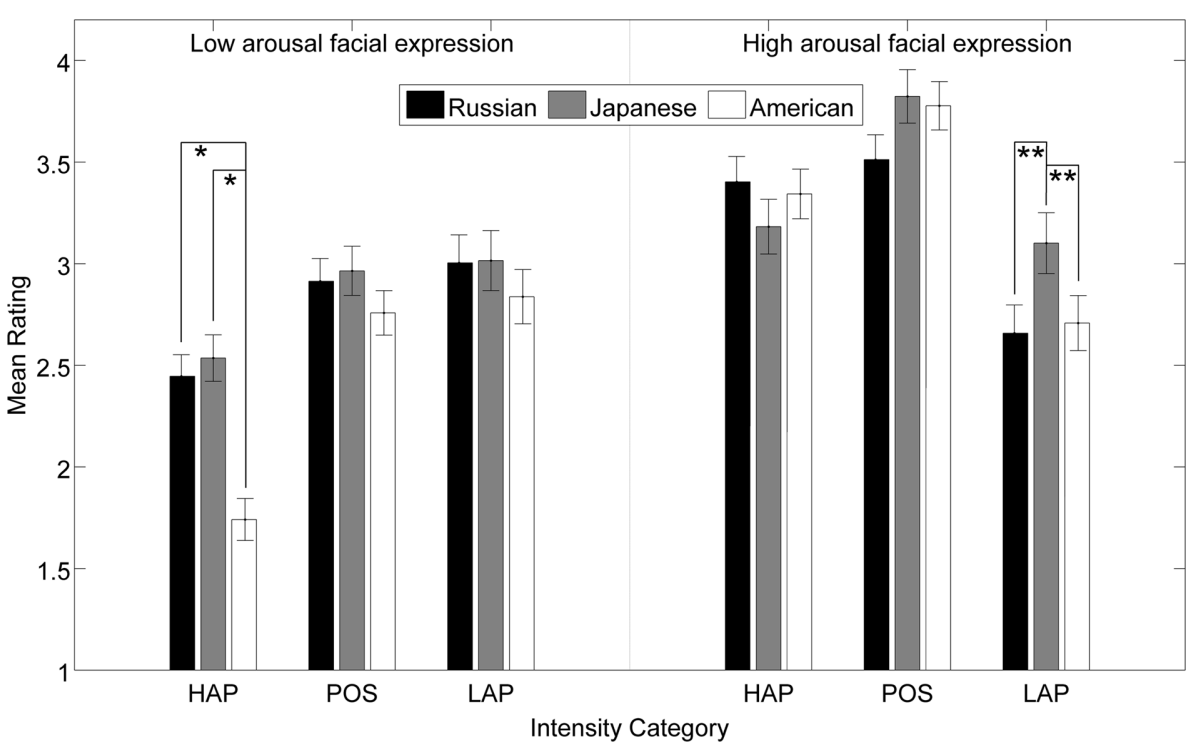

FIGURE 2 | Average ratings within each intensity category for advertisements showing LA and HA facial expressions across cultures. The figure shows mean ratings across cultures (Russian, Japanese, American) and emotion intensity categories (HAP, POS, LAP) for low and HA facial expressions. Significant cultural differences were obtained in the HAP intensity category for LA facial expressions and the LAP intensity category for HA facial expressions. ${ }^{*}=p<0.01$; ${ }^{*}=p<0.05$ indicating the presence of cultural differences in one or more of the rating categories. To identify exactly which cultural differences occurred, we conducted univariate follow-up tests of simple effects. These revealed significant cultural differences in mean ratings for the HAP intensity category after viewing LA advertisements $\left[F(2,58)=16.889, p<0.001, \eta^{2}=0.368\right]$. Post hoc pairwise comparisons (Fisher's LSD) revealed that American participants perceived advertisements with LA facial expressions as significantly less excited (HAP: 1.741) compared to Russian (2.446) and Japanese (2.535) participants (mean differences were significant at $p<0.001)$. Furthermore, a near-significant effect was obtained for mean ratings within the LAP intensity category after viewing HA advertisements $[F(2,58)=2.77, p=0.071$, $\left.\eta^{2}=0.087\right]$. Results from post hoc pairwise comparisons indicate that Japanese participants perceived HA images as significantly calmer (LAP: 3.101) compared to American (2.708) and Russian (2.658) participants [mean differences were significant at $p<0.05$ (JP vs. RU) and near-significant at $p=0.056$ (JP vs. US)]. All of these results were confirmed and extended by analyses of the ipsatized scores, with one minor exception in the post hoc comparisons (see Tables A2-A5 and Figure A1 in Appendix).

\section{DIFFERENCE SCORES}

Results from simple effects analyses indicate that cultures differ in their ratings of high and LA facial expressions. These results can be interpreted in two ways: they may indicate that (1) cultures perceive emotion intensity differentially, and (2) cultures have different ways of categorizing and labeling the perceived emotion. In order to investigate whether and to what extent these mechanisms contributed to the observed cross-cultural differences in affect intensity ratings, two independent types of difference scores were created. First, to address whether cultures perceive emotion intensity differently, difference scores between mean ratings for each arousal level within each intensity category were obtained. These scores reflect the degree to which participants from each culture were able to differentiate between differing intensity levels of facial expressions across intensity categories. For instance, we expected that participants from all cultures would rate HA facial expressions as more HAP than LA facial expressions (see also validation analysis in Table A1 in Stimulus Validation Analysis in Appendix), but that there would be significant cultural differences in the extent of such rating differences. Secondly, difference scores between mean ratings for each emotion intensity category within each arousal level were obtained. These difference scores reflect the degree to which participants from each culture differentiated between intensity categories, i.e., whether the observed emotion intensity (high or LA) was clearly classified into an intensity category across cultures. For instance, we expected that all cultures would rate HA facial expressions as more HAP than LAP, but that there would be significant cultural differences in the extent of such rating differences. Difference scores were created for each participant and entered into respective mixed Multivariate Analyses of Variance (MANOVA).

\section{Cultural differences in intensity perception}

To investigate the degree to which participants from each culture differentiated between the intensity of positive affect expressed by the model within each emotion intensity category, difference scores were obtained for each participant by subtracting mean ratings for LA from mean ratings for HA facial expressions of positive affect within each intensity category. Intensity difference scores reflecting the difference between ratings for HA 
compared to LA facial expressions were entered into a mixed two-way MANOVA with intensity category (HA-LA in HAP rating category, HA-LA in POS, HA-LA in LAP) as within-subjects factor and culture (American, Japanese, Russian) as betweensubjects factor. We observed a significant main effect of intensity category $\left[F(2,57)=67.529, p<0.001, \eta^{2}=0.703\right]$, indicating that rating categories, averaged over culture, showed significantly different mean scores from each other, with highest difference scores observed in the HAP rating category (1.068), followed by POS $(0.826)$ and LAP $(-0.13)$ (all post hoc pairwise comparisons were significant at $p<0.001)$. There was furthermore a significant main effect of culture $[F(2,58)=5.306$, $\left.p<0.01, \eta^{2}=0.155\right]$, indicating significantly greater difference scores, averaged over intensity categories, for American (0.831) compared to Japanese (0.403) and Russian (0.530) participants (post hoc pairwise comparisons were significant at $p<0.005$ for the contrast US vs. JP, and $p<0.05$ for the contrast US vs. RU). There was also an interaction between intensity category and culture $\left[F(4,114)=6.641, p<0.001, \eta^{2}=0.189\right]$. Because this interaction indicates that participants from the cultures included in the current study differentiated between high and LAP emotions expressed by the models to differing degrees (Figure 3), further analyses were conducted to examine this interaction and identify cultural differences in intensity perception.

To investigate general effects of culture on intensity difference scores, a between-subjects MANOVA was performed with culture as predictor variable and intensity difference scores in each intensity category as dependent variables (rating differences between high vs. LA facial expression across intensity categories were included as dependent variables: HA-LA_HAP, HA-LA_POS, HA-LA_LAP). Because the MANOVA indicated a significant effect of culture $\left[F(6,112)=6.443, p<0.001, \eta^{2}=0.257\right]$, indicating the presence of cultural differences for one or more of the intensity difference scores, we conducted univariate followup tests of simple effects to identify exactly for which intensity difference scores cultural differences occurred. Tests of simple effects indicated the presence of significant cultural differences in the HAP $\left[F(2,58)=13.842, p<0.001, \eta^{2}=0.323\right]$ and POS $\left[F(2,58)=3.204, p<0.05, \eta^{2}=0.099\right]$ intensity categories, as well as a marginally non-significant effect in the LAP intensity category $\left[F(2,58)=2.448, p=0.095, \eta^{2}=0.078\right]$. Pairwise comparisons (Fisher's LSD) revealed that in the HAP intensity category, US participants (1.602) differentiated between HA and LA images to a significantly larger degree compared to Russian $(0.957)$ and Japanese (0.646) participants [mean differences were significant at $p<0.005$ (US vs. RU) and $p<0.001$ (US vs. JP)]. Difference scores for American participants (1.019) were also significantly different from those of Russian (0.599) but not Japanese (0.859) participants in the POS intensity category (mean differences were significant at $p<0.05$ ). In the LAP intensity category, difference scores for Russian participants $(0.189)$ were significantly smaller than those of Japanese $(-0.262)$, but not American $(-0.021)$ participants (mean differences were significant at $p<0.05$; Figure 3 ). These results were fully confirmed by analyses of intensity difference scores based on ipsatized data, which are reported in Tables A6-A9 and Figure A2 in Appendix.

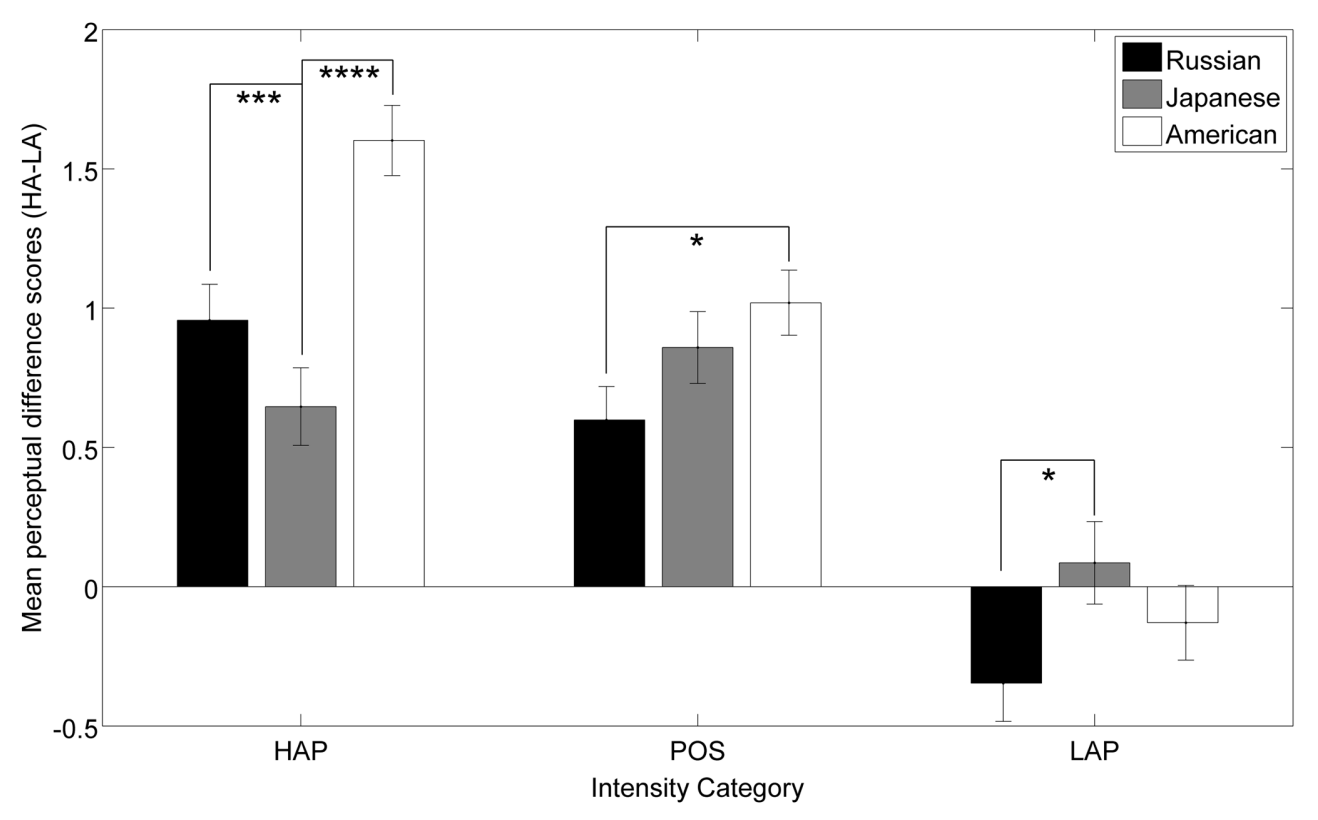

FIGURE 3 | Perceptual difference scores representing mean differences between ratings for high compared to LA facial expressions in each intensity category across cultures. American participants showed significantly greater differentiation between high and LA facial expressions in the HAP intensity category compared to Russian and Japanese participants.
In the POS intensity category, American participants differentiated between high and LA images to a significantly greater extent than Russian, but not Japanese participants. Finally, Russian participants perceived LA facial expressions as significantly lower arousal (LAP) compared to Japanese participants. ${ }^{* * *}=p<0.001 ;{ }^{* * *}=p<0.005 ;{ }^{* *}=p<0.01 ;{ }^{*}=p<0.05$. 


\section{Cultural differences in rating styles within each intensity category}

To investigate the degree to which participants from each culture differentiated between intensity categories for faces with the same arousal level, category difference scores were obtained for each participant by subtracting mean ratings within the LAP intensity category from both HAP and POS intensity categories (HAP-LAP; POS-LAP) and mean ratings in the POS intensity category from the HAP intensity category (HAP-POS) within each arousal level. These difference scores were entered into a mixed three-way MANOVA with category difference (HAP-LAP, HAPPOS, POS-LAP) and arousal level (high, low) as within-subjects factors and culture (American, Japanese, Russian) as betweensubjects factor. A significant main effect of category difference $\left[F(2,57)=74.747, p<0.001, \eta^{2}=0.724\right]$ was obtained, indicating that when averaged over the factors culture and arousal level, the greatest absolute difference was observed between HAP and POS ratings $(-0.516)$, followed by POS vs. LAP (0.404) and HAP vs. LAP $(-0.112)$ (all category differences were significantly different from each other at $p<0.001)$. A main effect of arousal level $\left[F(1,58)=118.301, p<0.001, \eta^{2}=0.671\right]$ was also observed, indicating that mean difference scores for HA faces (0.325) were greater than for LA faces $(-0.474)$ (post hoc comparisons showed significant differences at $p<0.001)$. There was also a near-significant main effect of culture $[F(2,58)=3.005$, $\left.p=0.057, \eta^{2}=0.094\right]$, indicating that mean difference scores of Japanese participants $(0.062)$ were significantly different from those of US participants $(-0.154 ; p<0.05)$, while a marginally non-significant difference was observed between Japanese and Russian participants $(-0.133 ; p=0.058)$. Culture interacted with the factors arousal level $\left[F(2,58)=9.322, p<0.001, \eta^{2}=0.243\right]$, indicating that for LA images, US participants' mean difference scores $(-0.731)$ were significantly smaller than those of Japanese $(-0.372 ; p<0.001)$ and Russian $(-0.320 ; p<0.05)$ participants, while for HA images, Russian participants' mean difference scores (0.054) were significantly smaller than those of Japanese $(0.496 ; p<0.001)$ and American $(0.423$; $p<0.005)$ participants. A further interaction between culture and category difference $\left[F(4,116)=3.614, p<0.01, \eta^{2}=0.113\right]$ was observed, indicating significant cultural differences for category differences averaged over arousal level, such that, for the contrast between HAP and LAP, Japanese participants' absolute mean difference scores $(0.93)$ were significantly smaller than those of US participants $(-0.231 ; p<0.05)$ and marginally non-significantly smaller compared to those of Russian participants $(-0.199, p=0.058)$. Furthermore, for the contrast between HAP and POS, Japanese participants' absolute mean difference scores $(-0.289)$ were significantly smaller than those of US $(-0.725 ; p<0.001)$ and RU $(-0.535 ; p<0.05)$ participants. A further two-way interaction was obtained between arousal level and category difference $\left[F(2,57)=67.529, p<0.001, \eta^{2}=0.703\right]$, indicating that all category differences for HA faces were significantly greater than category differences for LA faces (all post hoc pairwise comparisons were significant at $p<0.001)$. Importantly, there was a significant three-way interaction between culture, category difference, and arousal level $\left[F(4,114)=6.641, p<0.001, \eta^{2}=0.186\right]$. This interaction indicates that participants from different cultures differentiated between intensity categories as a function of arousal intensity of the facial expression of the models to different degrees.

To investigate general effect of culture on category difference scores as a function of arousal level, a between-subjects MANOVA was performed with culture as predictor variable and category difference scores in each arousal level as multiple dependent variables (rating differences between intensity category across arousal levels were included as dependent variables: rating differences between HAP and LAP ratings for LA facial expressions = HAP-LAP_LA, as well as HAP-POS_LA, POS-LAP_LA, HAP-LAP_HA, HAPPOS_HA, HAP-LAP_HA). Because the omnibus MANOVA indicated a significant effect of culture $[F(8,110)=5.214, p<0.001$, $\left.\eta^{2}=0.275\right]$, indicating the presence of significant cultural differences within at least one intensity category, we conducted univariate follow-up tests of simple effects to identify exactly for which category difference scores cultural differences occurred. Tests of simple effects indicate that cultural differences were present for LA facial expressions when contrasting (a) HAP to LAP ratings $\left[F(2,58)=5.066, p<0.01, \eta^{2}=0.149\right]$, and (b) HAP to $\mathrm{POS}$ ratings $\left[F(2,58)=9.115, p<0.001, \eta^{2}=0.239\right]$. Furthermore, cultural differences were present for HA facial expressions when contrasting (a) HAP and $\operatorname{LAP}[F(2,58)=6.944, p<0.005]$, and (b) HAP and POS $[F(2,58)=9.165, p<0.001]$. Post hoc tests (Fisher's LSD) were conducted to investigate these cultural differences. Post hoc tests revealed that American participants $(-1.096)$ differentiated between LAP and HAP affective dimensions to a much larger degree than Russian $(-0.558)$ and Japanese $(-0.480)$ participants when rating LA images [mean differences were significant at $p<0.05$ (US vs. RU) and $p<0.01$ (US vs. JP); Figure 4]. When rating HA facial expressions, Japanese participants $(0.081)$ differentiated between HAP and LAP affective dimensions significantly less than American (0.635) and Russian (0.745) participants [mean differences were significant at $p<0.005$ (JP vs. US) and $p<0.001$ (JP vs. RU)]. In fact, Japanese participants rated HA images equally on HAP and LAP dimensions, as indicated by a one sample $t$-test that tested whether difference scores were significantly different from $0[t(17)=0.65, p=0.523]$ (Figure 4). Further significant differences were obtained for US participants' difference scores $(-1.017)$ between HAP and POS when rating LA images compared to Russian $(-0.468)$ and Japanese $(-0.429)$ participants (mean differences were significant at $p<0.005)$. Finally, Russian participants $(-0.110)$ differentiated between HAP and POS to a smaller degree than Japanese $(-0.641)$ and American $(-0.434)$ participants when rating HA images (mean differences were significant at $p<0.005$ ). In fact, Russian participants rated HA images equally on HAP and POS dimensions, as indicated by a one sample $t$-test that tested whether difference scores were significantly different from $0[t(20)=-1.868, p=0.08]$. These results were fully confirmed by analyses of categorization difference scores based on ipsatized data, which are reported in Tables A10-A12 and Figure A3 in Appendix.

\section{DISCUSSION}

The present study investigated cultural differences in the perception of positive affect intensity in the context of advertisements for beauty products. As such, the present study addressed a previously 


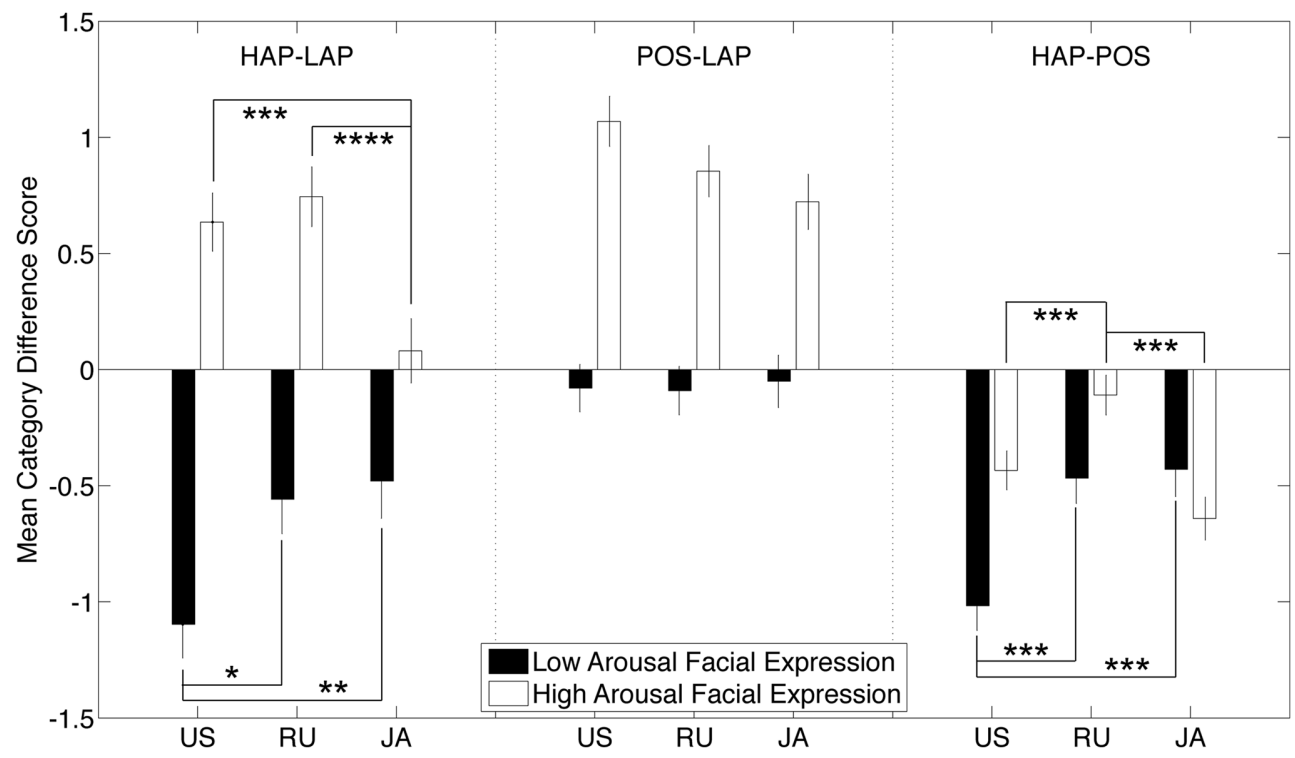

FIGURE 4 | Cultural differences in mean category difference scores as a function of arousal intensity of facial expressions. When contrasting HAP to LAP intensity categories, Japanese participants show significantly less differentiation for HA images compared to Russian and American participants. Similarly, American participants showed the greatest level of differentiation between HAP and LAP for LA facial expressions. When contrasting HAP to POS intensity categories, US participants show significantly more differentiation compared to Russian and Japanese participants. Finally, Russian participants show significantly less differentiation between HAP and POS rating categories for HA facial expressions. ${ }^{* * *}=p<0.001 ;{ }^{* *}=p<0.005 ;{ }^{* *}=p<0.01$;

${ }^{*}=p<0.05$ established limitation of past research on emotion perception in which participants are typically asked to judge affect in an artificial vacuum void of a particular context (Matsumoto, 2001). Thus, the use of advertisements as a contextual platform for ratings of facial expressions increased the ecological validity of the current study of emotion perception across cultures.

Findings from the current investigation indicate the presence of both cross-cultural differences, as well as agreement in the perception of positive affect intensity for images depicting both subtle (LA) and salient (HA) expressions of positive affect. Specifically, cultural differences of absolute ratings were obtained in (a) the LAP intensity category for HA images, and (b) the HAP intensity category for LA images. These findings reveal that American participants perceived HA images as significantly less calm than participants from the other two cultures, while Japanese participants perceived LA images as significantly more excited than participants from the other cultures. Cross-cultural agreement persisted in the POS and LAP intensity categories for LA images, as well as in POS and HAP intensity categories for HA images. Together, these results demonstrate the presence of cross-cultural differences in the perception of positive affect intensity at opposite ends of the arousal spectrum.

These results can be interpreted in two ways. Firstly, the findings may indicate that cultures perceive emotion intensity (i.e., high and LA) differentially. Secondly, they may be indicative of crosscultural differences in the categorization of perceived emotion intensities. In order to investigate these two plausible underlying mechanisms of cross-cultural differences in the ratings, two types of difference scores were obtained: (1) perceptual difference scores, and (2) categorical difference scores. Our results indicate cross-cultural differences in both underlying mechanisms, namely the perception of emotion intensity and categorization of emotion intensity.

Perceptual difference scores revealed specific similarities and differences in the perception of affect intensity across cultures. In particular, American participants showed significantly greater differentiation between high and LA facial expressions compared to Russian and Japanese participants in the HAP domain. Russian participants, on the other hand, differentiated between high and LA facial expressions to a significantly lower degree than American participants in the POS intensity category, and to a significantly greater degree compared to Japanese participants in the LAP intensity category. No differences were found between American and Japanese participants within these intensity categories. Taken together, these results indicate significant differences in the level of differentiation between high and LAP facial expressions across cultures. Specifically, American participants showed significantly higher differentiation between arousal levels of facial expressions in high and intermediate intensity categories (HAP, POS), while Russian participants also showed differentiation in the LA intensity category (LAP).

Category difference scores indicate cross-cultural variations in the way arousal intensities were categorized across cultures. Specifically, American participants rated LA images as significantly more low (LAP) and intermediate (POS) arousal than HA (HAP) compared to Russian and Japanese participants, and HA images as significantly more HA (HAP) than LA (LAP) compared to Japanese participants. These results indicate that American participants, relative to Japanese and Russian participants, distinctively categorized positive affect into low versus HA domains and that 
these domains are perceptually separate. Furthermore, the differences in ratings between HAP and LAP intensity categories were significantly smaller for Japanese compared to American and Russian participants, particularly for HA facial expressions. These results demonstrate that Japanese participants did not discriminate between high and LAP emotions to the same extent as did the American and Russian participants. Finally, Russian participants discriminated to a significantly lower degree between HAP and POS intensity categories than both Japanese and American participants, particularly for HA images. Our findings thus indicate that, while Americans showed the clearest differentiation between high and LA images across all intensity categories, Japanese and Russian participants deviated from such clear distinctions between affect intensity levels, such that the Japanese did not judge HA faces to be different in the HAP and LAP dimensions, while the Russians did not judge HA faces to be different in the HAP and POS dimensions. Taken together, these results indicate significant cross-cultural differences in the extent to which facial expressions are categorized into intensity categories.

Previous findings of cross-cultural differences in emotion valence perception may be applied to provide possible explanations for the present findings of differences in the perception of positive affect intensity. As previously suggested, cross-cultural differences in emotion perception may be due to cultural display rules imposed by cultural contexts (Matsumoto and Ekman, 1989), which can affect perception via modulating featural salience (Yuki et al., 2007) and emotion detection strategies (Damjanovic et al., 2010). For instance, while some cultures (e.g., American), may encourage the display of HAP emotions, display rules in other cultures (e.g., Japanese and Russian) may call for the inhibition of exaggerated display of affective expressions (Matsumoto et al., 1998, 2005; Matsumoto, 2001). Following this theory, individuals within the American cultural context are exposed to displays of affect with greater intensity and may therefore be able to recognize and categorize these emotions more readily compared to individuals from the Japanese cultural context. Recent findings from Damjanovic et al. (2010) lend support to this notion. The authors demonstrated cross-cultural difference in the expression of a facilitatory effect of other race targets for the detection of happy faces in the context of an affective visual search paradigm. In contrast to English participants, Japanese participants demonstrated such facilitatory effect of other race targets only when instructions specifically encouraged perceptual based rather than verbal labeling strategies. These results indicate that perceptually salient, HA smiles can lead to interference during the detection of affect for Japanese participants, as these are incongruent with Japanese display rules and therefore may not match the Japanese prototype emotion employed during visual search (see also Roberson et al., 2007). Findings from the present study indicate an analogous pattern in the domain of affect intensity perception for the Japanese participants, who showed the lowest level of differentiation between high and LAP emotions compared to other cultures (Figure 3) and, surprisingly, did not categorize HA facial expressions as either high or LA (Figure 4). This observation may be due to the fact that HA expressions do not match Japanese prototypical expressions, due to display rules that endorse deamplification and masking of positive emotional states (Matsumoto et al., 1998, 2008; Yuki et al., 2007) and therefore lead to perceptual interference as suggested previously (Damjanovic et al., 2010). Results from Japanese participants differ significantly from those of American and Russian participants. Specifically, compared to Japanese participants, American participants showed greater perceptual discrimination in the HA intensity domain, while Russian participants discriminated to a greater extent in the LA domain. Similarly, American participants categorized HA facial expressions as significantly more high and intermediate than LA when viewing HAP facial expressions. The current results, thus, extend previously established cross-cultural differences in the interpretation of affect valence among individuals from different cultural backgrounds by demonstrating a similar behavioral pattern in the perception of positive affect intensity.

Interestingly, in the present study, the pattern of results obtained from Russian participants fell between the American and Japanese participants for both perceptual difference scores and categorization difference scores. Specifically, Russian perceptual difference scores were different from American, but not Japanese participants in high and intermediate intensity categories. In the low intensity category, differences were obtained between Russian and Japanese, but not American, perceptual difference scores. Furthermore, when categorizing LAP images, Russian and Japanese participants had similar scores, while when categorizing HAP images, Russian participants scored more similar to the American participants. These results largely confirm our hypotheses about cultural agreement and differences between the three cultures included in the current investigation. Specifically, they show that Russian and Japanese participants perceive LA facial expressions similarly, as indicated by an absence of significant differences in mean ratings, as well as perceptual and category difference scores between these two cultures. For HA facial expressions, however, significant differences between Russian and Japanese participants were obtained in categorization difference scores.

The current results may be explained by previous findings indicating that emotion judgment is regulated not only by a universal and innate ability to recognize facial affect, but can also be modulated by certain culturally bestowed rules that vary according to sociocultural dimensions, such as Individualism/Collectivism (Matsumoto, 2001). Following this reasoning, we suggest that, due to cultural display rules, individuals within different cultural contexts are exposed to high intensity displays of positive affect to differing degrees, leading to differences in intensities of emotion prototypes. In the context of the current investigation, Americans are, on average, exposed to the highest intensity displays of positive affect relative to the other two cultures. While Russian and Japanese participants exert similar levels of control over emotional expressions, these cultures have also been shown to differ in their preferred method of controlling high intensity affect (Matsumoto et al., 2005). Specifically, Russians qualify their emotions to a greater degree than Japanese and Americans, while Japanese deamplify their emotions more than other cultures, leading to heterogeneous modifications of HA affect displays across these cultures (Matsumoto et al., 2005). Such exposure to different levels of high compared to low intensity emotion, as well as differentially modified affect, may affect prototype emotions for a given emotion category, as well as expected levels of deviation from such 
prototypes. Thus, one potential mechanism underlying the crosscultural differences in the perception of affect intensity observed in the current investigation can be attributed to cultural display rules. As such, these display rules lead to varying levels of exposure to high intensity positive affect, which, in turn, may create culturally biased prototype affect intensities and expected deviation levels.

The question of whether individuals from different cultural backgrounds interpret expressions of positive emotions in an analogous or dissimilar manner carries direct implications not only for psychological science, but for all aspects of intercultural communication. Interpersonal communication, particularly in an intercultural framework, draws extensively on non-verbal information, such as facial expressions, by providing valuable clues in navigating the interaction between interlocutors through their conveyance of emotional states (e.g., LaFrance and Mayo, 1978; Elfenbein and Ambady, 2002). However, variations in the recognition and, consequently, interpretation patterns of affective expressions across different cultural contexts may present obstacles in the process of intercultural communication. For example, in the present experiment the explicit distinction that the American participants showed between arousal intensities of positive affect differed from the rather vague discrimination of intensity levels among the Japanese. Specifically, while for Americans, LAP facial expressions were indicative of LAP emotions (e.g., calmness), the Japanese associated LAP facial expressions with HAP emotions (e.g., excitement) to a significantly greater degree than the other cultures (Figure 4). That is, the Japanese viewed high and LAP emotions as complementary, whereas the Americans viewed them as contradictory. These findings on affect intensity categorization parallel previously established patterns of cross-cultural differences in the interpretation of emotion valence (Uchida et al., 2004). As such, within the context of intercultural communication, a LA facial expression among the Japanese may be susceptible to a more inclusive and ambiguous interpretation than a LA facial expression among Americans.

Implications of findings of cross-cultural differences in affect perception and recognition that were revealed in the present study can also be observed in the field of advertising. For instance, even the slightest variations in the interpretation of emotional expressions featured in print advertisements could be detrimental to the delivery of affective messages that the marketers are trying to convey to consumers. Consequently, the interpretation of the ad content will influence the affective reactions of the consumers to the ads, which in turn, may affect the consumer response to the brand (Holbrook and Batra, 1987). This effect is particularly salient with advertisements for beauty products, since such advertisements typically involve close-ups of faces that are conveying positive affect of varying intensity levels. Thus, advertisers need to be aware of the variability of emotion perception in their international advertising campaigns that may be present among their audiences with different cultural backgrounds.

The main limitation of the current study is the exclusive use of Caucasian models as stimuli. The in-group advantage hypothesis, which was first proposed by Elfenbein and Ambady (2002) based on an extensive meta-analysis of cross-cultural data, suggests increased affect recognition accuracy when judge and poser are from the same cultural group. This raises the possibility that cultural differences in positive affect intensity perception obtained in the current study can, in part, be explained by an in-group advantage in emotion intensity recognition. While it is possible that the in-group advantage hypothesis extends to affect intensity perception, at least three factors mitigate the extent of potential in-group effects on emotion intensity ratings in the current investigation: (1) an in-group advantage only applies to differences between Asian and Western cultures, but does not extend to differences observed between Russian and American participants; (2) only expressions of positive affect were used in the current experiment. It has repeatedly been demonstrated that happiness is the most easily recognized basic emotion across both Asian and Western cultures (e.g., Matsumoto, 1992; Russell et al., 1993, for review see Russell, 1994). Furthermore, various studies have demonstrated a lack of an in-group advantage for happy faces. For instance, in a re-analysis of previously published data based on experiments with categorical emotion judgments (Matsumoto, 1992) and affect intensity ratings (Matsumoto and Ekman, 1989), Matsumoto (2002) showed that, for facial expressions of happiness, no differences between American and Japanese raters could be observed regardless of poser culture. Using a more conservative free-response method, Russell et al. (1993) found high recognition scores $(>80 \%)$ for happy facial expressions for raters from both Western and Asian cultures (Canadian, Greek, and Japanese), despite the fact that only Caucasian faces were used as stimuli in their study. Furthermore, a more recent experiment investigating cross-cultural differences in emotion recognition confidence (Beaupre and Hess, 2006), demonstrated an increased confidence when individuals judged emotion expressions of cultural in-group members, thereby confirming an in-group advantage for confidence. On the other hand, however, a general increase in emotion judgment-related confidence across all cultures was observed when judging happiness compared to other emotions. Taken together, these findings demonstrate a weakening of an ingroup advantage in recognition and confidence when positively valenced facial expressions are judged. One explanation for these results can be found in Matsumoto et al. (2005), who showed that across American, Japanese, and Russian nationals, the emotion that is least affected by display rules is happiness, suggesting an increased familiarity across cultures with relatively unmodified displays of happiness compared to other emotions. (3) Familiarity with the poser culture has been demonstrated to reduce an ingroup advantage in emotion recognition (Elfenbein and Ambady, 2002, 2003; see also O’Toole et al., 1994; O’Toole et al., 1996). In the present study, ratings of affect intensity were conducted in the context of beauty advertisements. Within this context, Japanese as well as Russian and American participants are highly accustomed to viewing Western models due to a strong prevalence of Caucasian models in international and national marketing campaigns in the beauty industry (e.g., Mueller, 1992; Cutler et al., 1995; Moeran, 2002; Barnes and Yamamoto, 2008). Therefore, a weakening of the cultural in-group advantage can be assumed within the context of advertising. Taken together, these points mitigate potential influences of an in-group advantage in the current investigation.

The above discussion raises interesting questions as to whether and to what extent the in-group advantage hypothesis generalizes 
to the perception of affect intensity, which may be addressed in future research endeavors. Foremost among those is an investigation of an in-group advantage in affect intensity perception in both positive and negative valence domains. To this end, posers and raters from each culture would need to be included in one study, ideally using a fully balanced design (Matsumoto, 2002).

\section{CONCLUSION}

In the past few decades, psychological research has attested to the existence of subtle differences in the perception of emotions across cultures, thus solidifying the significance of culture in all aspects of the emotional mechanism (Matsumoto, 1992). The present study supported and extended previous findings by revealing cross-cultural differences in the perception of positive affect intensity among American, Japanese, and Russian female participants within the context of advertising. Findings indicated that American participants discriminated between high and LAP facial expressions to a greater extent than the Russians and the Japanese in the HA domain, and categorized high and LA facial expressions differently compared to the other cultures. The Japanese participants, on the other hand, showed the lowest discrimination of positive affect intensities, particularly for HA facial expressions. The Russian participants demonstrated similar affect perception patterns to both Americans (for HA facial expressions) and the Japanese (for LA facial expressions). The findings of cross-cultural differences within the positive affect intensity continuum obtained by the present study are intriguing, because they parallel previously established cross-cultural differences in the interpretation of positive and negative emotions. In other words, the previously established comprehensive model of cultural variability of emotion perception has now also been observed within the positive affect intensity continuum. The observed cross-cultural

\section{REFERENCES}

Barnes, B. R., and Yamamoto, M. (2008). Exploring international cosmetics advertising in Japan. J. Mark. Manage. 24, 299-316.

Beaupre, M. G., and Hess, U. (2006). An ingroup advantage for confidence in emotion recognition judgments: The moderating effect of familiarity with the expression of outgroup members. Pers. Soc. Psychol. Bull. 32, 16-26.

Biehl, M., Matsumoto, D., Ekman, P., Hearn, V., Heider, K., and Kudoh, T. (1997). Matsumoto and Ekman's Japanese and Caucasian facial expressions of emotion (JACFEE): reliability data and cross-national differences. J. Nonverb. Behav. 21, 3-21.

Buck, R. (1988). Human Motivation and Emotion, 2nd Edn. New York: Wiley.

Calvo, M. G., and Nummenmaa, L. (2008). Detection of Emotional Faces: Salient physical features guide effective visual search. J. Exp. Psychol. Gen. 137, 471-494.
Chan, D. W. (1985). Perception and judgment of facial expressions among the Chinese. Int. J. Psychol. $20,681-692$.

Chan, W. (2003). Analyzing ipsative data in psychological research. Behaviormetrika 40, 99-121.

Chen, C., Lee, S. Y., and Stevenson, H. W. (1995). Response style and cross-cultural comparisons of rating scales among east Asian and north American students. Psychol. Sci. 6, 170-175.

Cutler, B. D., Javalgi, R. G., and White, D. S. (1995). The westernization of Asian print advertising. J. Int. Consum. Mark. 7, 23-37.

Damjanovic, L., Roberson, D., Athanasopoulos, P., Kasai, C., and Dyson, M. (2010). Searching for happiness across cultures. J. Cogn. Cult. 10, 85-107.

Ekman, P., and Friesen, W. V. (1969). The repertoire of non-verbal behavior: categories, origins, usage, and coding. Semiotica 1, 49-98.

differences were attributed to variations in display rules that regulate exposure to different intensity levels of positive affect across cultures and thereby modulate emotion prototypes and expected deviations from these.

Furthermore, the present study addressed a previous limitation of perception research in two ways. Firstly, perception of emotion was investigated within a context ubiquitous in all three cultures, namely that of beauty advertising, thus providing a more naturalistic setting for the study of affect perception. Secondly, a novel instrument was employed to study the perception of positive affect in the context of beauty ads, which allowed participants to rank perceived affect in various intensity domains, rather than in the valence domains alone.

The current findings underline differences in emotion perception and categorization across cultures, which carry direct implications for all facets of communication, including advertising. A keen awareness of variations in sociocultural rules and their influences on affective mechanisms therefore becomes imperative for successful communication within a multicultural framework in a wide variety of situations that range from interpersonal settings to the effective execution of global advertising campaigns.

\section{ACKNOWLEDGMENTS}

We would like to thank Jacqueline Wasilewski for helpful comments on an earlier version of this manuscript. We also thank Natalia Meliksiatova and Nahoko Hirano for help with translating the questionnaires. Marianna Pogosyan was supported by the Center of Excellence (COE) Grant from the Japanese Ministry of Education for Peace, Security, and Conviviality. Jan B. Engelmann gratefully acknowledges support from the Mercator Foundation Switzerland, the NCCR Affective Sciences, and the Neurochoice project of SystemsX, as well as the research priority program at the University of Zurich "Foundations of Human Social Behavior."

Ekman, P., and Friesen, W. V. (1978). The Facial Action Coding System: A Technique for the Measurement of Facial Movement. Palo Alto, CA: Consulting Psychologists Press.

Ekman, P., Friesen, W. V., and Ellsworth, P. (1972). Emotion in the Human Face: Guidelines for Research and an Integration of Findings. New York: Pergamon Press.

Ekman, P., Friesen, W. V., O'Sullivan, M., Chan, A., Diacoyanni-Tarlatzis, I., and Heider, K. (1987). Universals and cultural differences in the judgments of facial expressions of emotion. J. Pers. Soc. Psychol. 53, 712-717.

Ekman, P., and Heider, K. G. (1988). The universality of a contempt expression: A replication. Motiv. Emot. 12, 303-308.

Elfenbein, H. A., and Ambady, N. (2002). On the universality and cultural specificity of emotion recognition: a meta-analysis. Psychol. Bull. 128, 203-235.
Elfenbein, H. A., and Ambady, N. (2003). When familiarity breeds accuracy: cultural exposure and facial emotion recognition. J. Pers. Soc. Psychol. 85, 276-290.

Fischer, R. (2004). Standardization to account for cross-cultural response bias. a classification of score adjustment procedures and review of research in JCCP. J. Cross Cult. Psychol. 35, 263-282.

Grammer, K., and Eibl-Eibesfeldt, I. (1993). "Emotionpsychologie im Kulturenvergleich [emotion psychology in cultural comparisons]," in Kulturvergleichende Psychologie Eine Eifuhrung ed A. Thomas (Gottingen: Hogrefe), 289-322.

Haidt, J., and Keltner, D. (1999). Culture and facial expression: openended methods find more expressions and a gradient of recognition. Cogn. Emot. 13, 225-266.

Heine, S. J., Lehman, D. R., Markus, H. R., and Kitayama, S. (1999). Is there a universal need for positive selfregard? Psychol. Rev. 106, 766-794. 
Hicks, L. E. (1970). Some properties of ipsative, normative and forcedchoice normative measures. Psychol. Bull. 74, 167-184.

Hofstede, G. (1980). Cultures' Consequences: International Differences in Work-Related Values. Beverly-Hills, CA: Sage.

Holbrook, M. B., and Batra, R. (1987). Assessing the role of emotions as mediators of consumer responses to advertising. J. Consum. Res. 14, 404-420.

Izard, C. E. (1971). The Face of Emotion. New York: Appleton-CenturyCrofts.

Ko, S.-G., Lee, T.-H., Yoon, H.-Y., Kwon, J.-H., and Mather, M. (2011). How does context affect assessments of facial emotion? The role of culture and age. Psychol. Aging 26, 48-59.

Krumhuber, E. G., and Scherer, K. R. (2011). Affect bursts: dynamic patterns of facial expression. Emotion 11, 826-841.

LaFrance, M., and Mayo, C. (1978). Cultural aspects of non-verbal communication. Int. J. Intercult. Relat. 2, 71-89.

LeDoux, J. E. (1987). "Emotion," in Handbook of physiology, Vol. 1, The nervous system, ed V. Mount Castle (Bethesda, MD: American Physiological Society), 419-459.

Leung, J. P., and Singh, N. N. (1998). Recognition of facial expressions of emotion by Chinese adults with mental retardation. Behav. Modif. 22, 205-216.

Levin, D. T. (2000). Race as a visual feature: using visual search and perceptual discrimination tasks to understand face categories and the crossrace recognition deficit. J. Exp. Psychol. Gen. 129, 559-574.

Mandal, M. K., Saha, G. B., and Palchoudhury, S. (1986). A crosscultural study on facial affect. $J$. Psychol. Res. 30, 140-143.

Markus, H. R., and Kitayama, S. (1991). Culture and the self: implications for cognition, emotion, and motivation. Psychol. Rev. 98, 224-253.

Masuda, T., Ellsworth, P., Mesquita, B., Leu, J., Tanida, S., and Van de Veerdonk, E. (2008). Placing the face in context: cultural differences in the perception of facial emotion. J. Pers. Soc. Psychol. 94, 365-381.

Matsumoto, D. (1990). Cultural similarities and differences in display rules. Motiv. Emot. 14, 195-214.

Matsumoto, D. (1992). AmericanJapanese cultural differences in the recognition of universal facial expressions. J. Cross Cult. Psychol.23, 72-84.
Matsumoto, D. (1993). Ethnic differences in affect intensity, emotion judgments, display rules, and self-reported emotional expression. Motiv. Emot. 17, 107-123.

Matsumoto, D. (2001). Culture and Emotion. Oxford: Oxford University Press.

Matsumoto, D. (2002). Methodological requirements to test a possible ingroup advantage in judging emotions across cultures: comments on Elfenbein and Ambady and evidence. Psychol. Bull. 128, 236-242.

Matsumoto, D., Consolacion, T., Yamada, H., Suzuki, R., Franklin, B., Paul, S., Ray, R., and Uchida, H. (2002). American-Japanese cultural differences in judgments of emotional expressions of different intensities. Cogn. Emot. 16, 721-747.

Matsumoto, D., and Ekman, P. (1989). American-Japanese cultural differences in intensity ratings of facial expressions of emotion. Motiv. Emot. 13, 143-157.

Matsumoto, D., Kasri, F., and Kooken, K. (1999). American-Japanese cultural differences in judgments of expression intensity and subjective experience. Cogn. Emot. 13, 201-218.

Matsumoto, D., Takeuchi, S., Andayani, S., Kouznetsova, N., and Krupp, D. (1998). The contribution of individualism vs. collectivism to crossnational differences in display rules. Asian J. Soc. Psychol. 1, 147-165.

Matsumoto, D., Yoo, S. H., Fontaine, J., Anguas-Wong, A. M., Arriola, M., Ataca, B., Bond, M. H., Boratav, H. B., Breugelmans, S. M., Cabecinhas, R., Chae, J., Chin, W. H., Comunian, A. L., DeGere, D. N., Djunaidi, A., Fok, H. K., Friedlmeier, W., Ghosh, A., Glamcevski, M., Granskaya, J. V., Groenvynck, H. Harb, C., Haron, F., Joshi, R., Kakai, H., Kashima, E., Khan, W., Kurman, J., Kwantes, C. T., Mahmud, S. H., Mandaric, M., Nizharadze, G., Odusanya, J. O. T., Ostrosky-Solis, F., Palaniappan, A. K., Papastylianou, D., Safdar, S., Setiono, K., Shigemasu, E., Singelis, T. M., Polackova Solcova, Iva, Spieß, E., Sterkowicz, S., Sunar, D., Szarota, P., Vishnivetz, B., Vohra, N., Ward, C., Wong, S., Wu, R., Zebian, S., and Zengeya, A. (2008). Mapping expressive differences around the world: The relationship between emotional display rules and Individualism vs. Collectivism. J. Cross Cult. Psychology 39, 55-74.

Matsumoto, D., Yoo, S. H., Hirayama, S., and Petrova, G. (2005). Development and initial validation of a measure of display rules: the display rule assessment inventory (DRAI). Emotion 5, 23-40.

Maxwell, S. E., and Delaney, H. D. (2000). Designing Experiments and Analyzing Data: A Model Comparison Perspective. Mahwah, NJ: Lawrence Erlbaum Associates.

Michel, C., Rossion, B., Han, J., Chung, C.S., and Caldara, R. (2006). Holistic processing is finely tuned for faces of one's own race. Psychol. Sci. 17, 608-615.

Moeran, B. (2002). "Japanese advertising discourse: reconstructing images," in Exploring Japaneseness: On Japanese Enactments of Culture and Consciousness, Civic Discourse for the Third Millennium series, ed R. T. Donahue (London: Greenwood Publishing Group), 386-398.

Mueller, B. (1992). Standardisation vs. specialisation: an examination of westernisation in cJapanese advertising. J. Advert. Res. 32, 15-24.

Nisbett, R. E., Peng, K. P., Choi, I., and Norenzayan, A. (2001). Culture and systems of thought: holistic versus analytic cognition. Psychol. Rev. 108 291-310.

O’Toole, A. J., Deffenbacher, K. A., Valentin, D., and Abdi, H. (1994). Structural aspects of face recognition and the other-race effect. Mem Cognit. 22, 208-224.

O'Toole, A. J., Peterson, J., and Deffenbacher, K. A. (1996). An 'other-race effect' for categorizing faces by sex. Perception 25, 669-676.

Roberson, D., Damjanovic, L., and Pilling, M. (2007). Categorical perception of facial expressions: evidence for a 'category adjustment' model. Mem. Cognit. 35, 1814-1829.

Russell, J. A. (1991). Culture and the categorization of emotions. Psychol. Bull. 110, 426-450.

Russell, J. A. (1994). Is there universal recognition of emotion from facial expression? A review of the crosscultural studies. Psychol. Bull. 115, 102-141.

Russell, J. A., Suzuki, N., and Ishida, N. (1993). Canadian, Greek, and Japanese freely produced emotion labels for facial expressions. Motiv. Emot. 17, 337-351.

Safdar, S., Friedlmeier, W., Matsumoto, D., Yoo, S. H., Kwantes, C., Kakai, H. and Shigemasu, E. (2009). Variations of emotional display rules within and across cultures: a comparison between Canada, USA, and Japan. Can. J. Behav. Sci. 41, 1-10.

Scherer, K. R., and Ellgring, H. (2007). Are facial expressions of emotion produced by categorical affect programs or dynamically driven by appraisal. Emotion 7, 113-130.

Thorson, E. (1990). "Consumer processing of advertising in current issues and research in advertising," in Current Research and Issues in Advertising, eds J. H. Leigh and C. Martin (Ann Arbor, MI: University of Michigan School of Business), 197-230.

Toner, H. L., and Gates, G. R. (1985). Emotional traits and recognition of facial expression of emotion. J. Nonverb. Behav. 9, 48-66.

Tsai, J. L., Knutson, B., and Fung, H. H. (2006). Cultural variation in affect valuation. J. Pers. Soc. Psychol. 90, 288-307.

Uchida, Y., Norasakkunkit, V., and Kitayama, S. (2004). Cultural constructions of happiness: theory and empirical evidence. J. Happiness Stud. 5, 223-239.

Yik, M., and Russell, J. A. (2003). Chinese affect circumplex: I. Structure of recalled momentary affect. Asian J. Soc. Psychol. 6, 185-200

Yrizarry, N., Matsumoto, D., and Wilson Cohn, C. (1998). American and Japanese multi-scalar intensity ratings of universal facial expressions of emotion. Motiv. Emot. 22, 315-327.

Yuki, M., Maddux, W. W., and Masuda, T. (2007). Are the windows to the soul the same in the East and West? Cultural differences in using the eyes and mouth as cues to recognize emotions in Japan and the United States. J. Exp. Soc. Psychol. 43, 303-311.

Conflict of Interest Statement: The authors declare that the research was conducted in the absence of any commercial or financial relationships that could be construed as a potential conflict of interest.

Received: 28 July 2011; accepted: 16 October 2011; published online: 07 November 2011.

Citation: Pogosyan $M$ and Engelmann JB (2011) Cultural differences in affect intensity perception in the context of advertising. Front. Psychology 2:313. doi: 10.3389/fpsyg.2011.00313

This article was submitted to Frontiers in Cultural Psychology, a specialty of Frontiers in Psychology.

Copyright (C) 2011 Pogosyan and Engelmann. This is an open-access article subject to a non-exclusive license between the authors and Frontiers Media SA, which permits use, distribution and reproduction in other forums, provided the original authors and source are credited and other Frontiers conditions are complied with. 


\section{APPENDIX STIMULUS VALIDATION ANALYSIS}

Table A1 | Results from post hoc stimulus validation analysis.

\begin{tabular}{|c|c|c|c|c|c|c|}
\hline Model pairs & Mean difference & SEM difference & $\boldsymbol{t}$ & df & \multicolumn{2}{|c|}{$p$-Value } \\
\hline Pair 2: HAP_H_2 - HAP_L_2 & 0.311475409803 & 0.126094203572 & 2.470 & 60 & $0.016^{*}$ & $0.008 * *$ \\
\hline Pair 3: HAP_H_3 - HAP_L_3 & 1.644808743230 & 0.154446118991 & 10.650 & 60 & $0.000^{*}$ & $0.000 * *$ \\
\hline Pair 4: HAP_H_4 - HAP_L_4 & 1.207650273262 & 0.154504109990 & 7.816 & 60 & $0.000^{*}$ & $0.000 * *$ \\
\hline Pair 6: HAP_H_6 - HAP_L_6 & 1.125683060098 & 0.141155766739 & 7.975 & 60 & $0.000^{*}$ & $0.000 * *$ \\
\hline Pair 7: HAP_H_7 - HAP_L_7 & 0.841530054672 & 0.161129618446 & 5.223 & 60 & $0.000^{*}$ & $0.000 * *$ \\
\hline Pair 8: HAP_H_8 - HAP_L_8 & 1.273224043689 & 0.130504647686 & 9.756 & 60 & $0.000^{*}$ & $0.000 * *$ \\
\hline Pair 9: HAP_H_9 - HAP_L_9 & 0.612021857967 & 0.173569323714 & 3.526 & 60 & $0.001 *$ & $0.000 * *$ \\
\hline Pair 10: HAP_H_10 - HAP_L_10 & 1.562841530066 & 0.133501938860 & 11.707 & 60 & $0.000^{*}$ & $0.000 * *$ \\
\hline Pair 11: HAP_H_11 - HAP_L_11 & 0.912568306016 & 0.166921779134 & 5.467 & 60 & $0.000^{*}$ & $0.000^{* *}$ \\
\hline
\end{tabular}

${ }^{*}$ All tests survived a FDR corrected (-level of 0.05, **all tests survived a FDR corrected (-level of 0.01. Paired differences between HAP ratings for high compared to $L A$ facial expressions for each model pair across all participants independent of culture are shown. Analyses tested the directional hypothesis that images classified as HA facial expressions were indeed perceived as significantly higher arousal (HAP) than LA facial expressions for each model pair. In order not to bias the current validation analysis toward one culture, we included results from all participants, independent of culture. Results show significantly higher HAP ratings for HA faces compared to $L A$ faces for all model pairs included in the current investigation. Results survived corrections for multiple comparisons $\left(N_{\text {tests }}=11\right)$ using false discovery rate (FDR) correction at an ( level of 0.01 for one-tailed tests (although the tests were directional, two-tailed results are also reported, which survived an FDR correction at an (level of 0.05).

\section{ANALYSES OF IPSATIZED DATA}

In order to control for potential cultural differences in rating style and response set bias (e.g., Hofstede, 1980; Chen et al., 1995), all data were standardized and reanalyzed. Specifically, to control for two types of bias that could systematically affect emotion ratings across culture, namely (a) an extreme response style that is characterized by a tendency to use the endpoints of a scale and (b) acquiescence response bias that is characterized by a shift to either end of a scale, we employed ipsatization as our method of standardizing raw scores. Ipsatization was implemented within-subject by subtracting each participant's grand mean for all 198 items (22 images $\times 9$ ratings) from his or her raw scores for each item and dividing this difference by the standard deviation across all items $\left[y_{\text {ips }}=(x-(\right.$ individual $) /($ individual $]$ (e.g., Yik and Russell, 2003; see also Hicks, 1970; Hofstede, 1980; Chan, 2003; Fischer, 2004). Ipsatized individual scores were then averaged across models to create mean scores in each rating category (HAP, POS, LAP). Results from this re-analysis of the data supported findings obtained in analyses of the raw data reported in the main paper. Since the logic of the re-analysis of the data follows that outlined in the main paper, each analysis step is only briefly summarized in the header of the table summarizing each model.

Table A2 | Omnibus MANOVA for mean ipsatized scores.

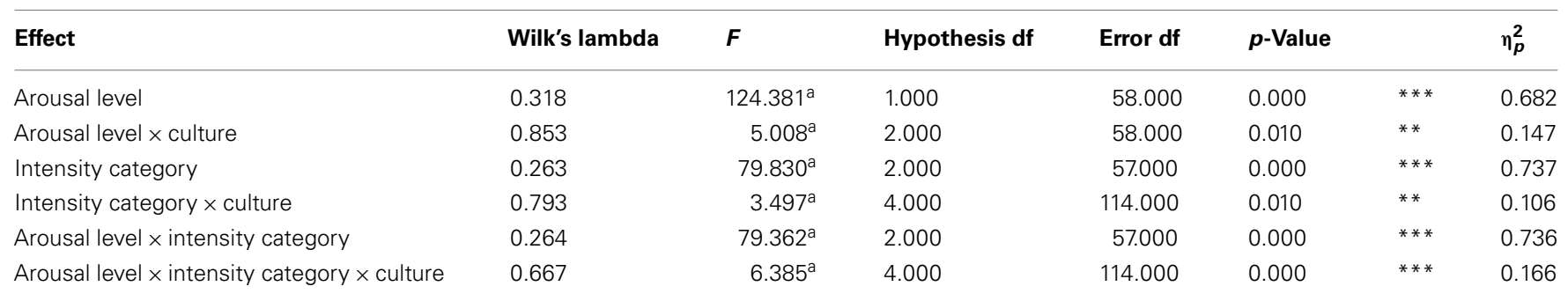

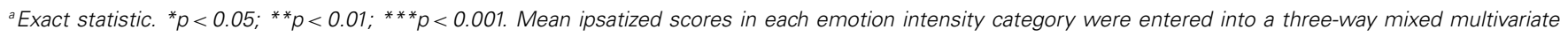

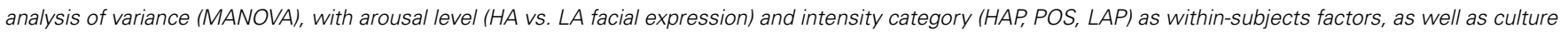

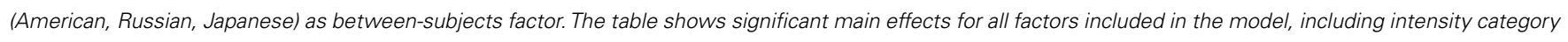

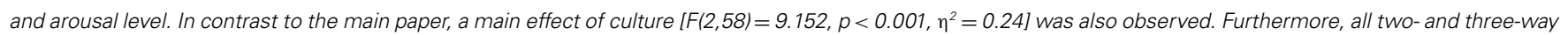
interactions were significant. 
Table A3 | Between-subjects MANOVA testing the effect of culture on mean ipsatized ratings.

\begin{tabular}{lllllll}
\hline Effect & Wilk's lambda & $\boldsymbol{F}$ & Hypothesis df & Error df & $\boldsymbol{p}$-Value & $\eta_{\boldsymbol{p}}^{\mathbf{2}}$ \\
\hline Culture & 0.360 & $5.885^{\mathrm{a}}$ & 12.000 & 106.000 & 0.000 & $* * *$ \\
\hline
\end{tabular}

${ }^{a}$ Exact statistic. ${ }^{*} p<0.05 ;{ }^{*} p<0.01 ;{ }^{* *} p<0.001$. Investigations of the nature of the three-way interaction obtained in the omnibus MANOVA focused on identifying the effects of culture on mean ipsatized ratings and followed the following sequence: first, to investigate general effects of culture on ratings within each intensity category as a function of arousal level, a between-subjects MANOVA was performed with culture as predictor variable and ratings across intensity category and arousal level as multiple dependent variables [HAP_LA, POS_LA, LAP_LA, HAP_HA, POS_HA, LAP_HA]. As shown in the table, a significant effect of culture was obtained.

Table A4 | Univariate tests of simple effects.

\begin{tabular}{|c|c|c|c|c|c|c|c|}
\hline Source & Dependent variable & Hypothesis df & Error df & Mean square (error) & $\boldsymbol{F}$ & $p$-Value & $\eta_{p}^{2}$ \\
\hline \multirow[t]{4}{*}{ Corrected model, culture } & HAP_L & 2 & 58 & $1.420(0.114)$ & 12.407 & $0.000 * * *$ & 0.300 \\
\hline & POS_L & 2 & 58 & $0.026(0.090)$ & 0.294 & 0.747 & 0.010 \\
\hline & HAP_H & 2 & 58 & $0.565(0.097)$ & 5.841 & $0.005^{* *}$ & 0.168 \\
\hline & POS_H & 2 & 58 & $0.491(0.058)$ & 8.519 & $0.001 * *$ & 0.227 \\
\hline
\end{tabular}

${ }^{*} p<0.05 ;{ }^{*} p<0.01 ;{ }^{* *} p<0.001$. Given the significant effect of culture obtained in the between-subjects MANOVA reported in Table A3, indicating the presence of cultural differences in at least one of the rating categories, univariate tests of simple effects were conducted to identify rating categories that show a significant effect of culture. Results that confirm those reported in the main paper are shown in bold, but additional significant effects were also obtained after ipsatization and include effects of culture on ratings of HA images in the HAP and POS rating categories. 
Table A5 | Post hoc comparisons of ipsatized scores were conducted following the logic outlined in the main paper.

\begin{tabular}{|c|c|c|c|c|c|}
\hline Dependent variable & (I) Culture & (J) Culture & Mean difference (I-J) & SE & $p$-Value \\
\hline \multirow[t]{5}{*}{ HAP_L } & $J P$ & $\mathrm{RU}$ & -0.01734912601 & 0.108655681689 & 0.874 \\
\hline & & US & $0.43972486946^{*}$ & 0.107509895556 & $0.000 * * *$ \\
\hline & & US & $0.45707399547^{*}$ & 0.103199900190 & $0.000 * * *$ \\
\hline & US & JP & $-0.43972486946^{*}$ & 0.107509895556 & $0.000 * * *$ \\
\hline & & RU & $-0.45707399547^{*}$ & 0.103199900190 & $0.000 * * *$ \\
\hline \multirow{5}{*}{ HAP_H } & & US & $-0.31286846172^{*}$ & 0.098831390842 & $0.002 * *$ \\
\hline & $R U$ & $J P$ & $0.28008737204^{*}$ & 0.099884685858 & $0.007^{* *}$ \\
\hline & & US & -0.03278108968 & 0.094869310566 & 0.731 \\
\hline & US & $J P$ & $0.31286846172^{*}$ & 0.098831390842 & $0.002 * *$ \\
\hline & & RU & 0.03278108968 & 0.094869310566 & 0.731 \\
\hline \multirow{2}{*}{ POS_H } & US & $J P$ & $0.15709977785^{*}$ & 0.076287651886 & $0.044^{*}$ \\
\hline & & $\mathrm{RU}$ & $0.30209531973^{*}$ & 0.073229334096 & $0.000 * * *$ \\
\hline \multirow[t]{6}{*}{ LAP_H } & $J P$ & RU & $0.28551301603^{*}$ & 0.110708936201 & $0.012^{*}$ \\
\hline & & US & 0.11993161801 & 0.109541498273 & 0.278 \\
\hline & $R U$ & $J P$ & $-0.28551301603^{*}$ & 0.110708936201 & $0.012^{*}$ \\
\hline & & US & -0.16558139802 & 0.105150057396 & 0.121 \\
\hline & US & $J P$ & -0.11993161801 & 0.109541498273 & 0.278 \\
\hline & & $R U$ & 0.16558139802 & 0.105150057396 & 0.121 \\
\hline
\end{tabular}

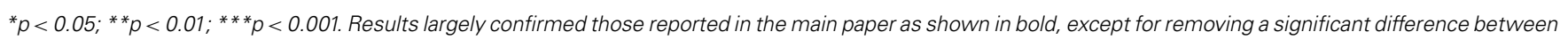

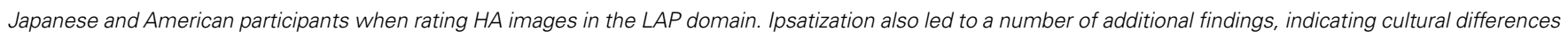
in further rating categories for HA faces compared to the main paper. These include significant cultural differences when HA images were rated in (1) the HAP domain,

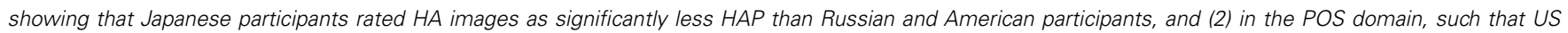
participants rated HA images as significantly more POS than Japanese and Russian participants.

\section{ANALYSES OF INTENSITY DIFFERENCES SCORES BASED ON IPSATIZED DATA}

Table A6 | Omnibus MANOVA for intensity difference scores of ipsatized data.

\begin{tabular}{|c|c|c|c|c|c|c|}
\hline Effect & Wilk's lambda & $\boldsymbol{F}$ & Hypothesis df & Error df & $p$-Value & $\eta_{p}^{2}$ \\
\hline Intensity_Category ${ }^{*}$ Culture & 0.667 & $6.385^{a}$ & 4.000 & 114.000 & $0.000 * * *$ & 0.183 \\
\hline
\end{tabular}

${ }^{a}$ Exact statistic. ${ }^{*} p<0.05 ;{ }^{*} p<0.01 ; * * * 0.001$. Intensity difference scores of ipsatized data were entered into a mixed two-way MANOVA with intensity category (HA-LA) facial expression in HAP rating category, HA-LA_POS, HA-LA_LAP) as within-subjects factor and culture (American, Japanese, Russian) as between-subjects factor. Similar to results reported in the main paper, results based on ipsatized data yielded significant main effects of intensity category and culture $[F(2,58)=5.008$, $\left.p=0.01, \eta^{2}=0.147\right]$, as well as an interaction between intensity category and culture. 
Table A7 | Between-subjects MANOVA to investigate the effects of culture on intensity difference scores from ipsatized data.

\begin{tabular}{lllllll}
\hline Effect & Wilk's lambda & $\boldsymbol{F}$ & Hypothesis df & Error df & $\boldsymbol{p}$-Value & $\boldsymbol{\eta}_{\boldsymbol{p}}^{\mathbf{2}}$ \\
\hline Culture & 0.562 & $6.244^{\mathrm{a}}$ & 6.000 & 112.000 & $0.000^{* * *}$ & 0.251
\end{tabular}

${ }^{a}$ Exact statistic. ${ }^{*} p<0.05 ;{ }^{*} p<0.01 ;{ }^{* *} p<0.001$. Investigations of the nature of the two-way interaction obtained in the omnibus MANOVA focused on identifying the effects of culture on ipsatized difference scores and followed the following sequence: first, to investigate general effects of culture on intensity difference scores within each intensity category, a between-subjects MANOVA was performed with culture as predictor variable and difference scores across intensity category as multiple dependent variables [HA-LA_HAP (Rating differences in the HAP domain for high vs. LA facial expression), HA-LA_POS, HA-LA_LAP]. As shown in the table, a significant effect of culture was obtained.

Table A8 | Univariate tests of simple effects of intensity difference scores from ipsatized data.

\begin{tabular}{|c|c|c|c|c|c|c|c|}
\hline Source & Dependent variable & Hypothesis df & Error df & Mean square (error) & $\boldsymbol{F}$ & $p$-Value & $\eta_{p}^{2}$ \\
\hline \multirow[t]{2}{*}{ Corrected model, culture } & HAP_HvL & 2 & 58 & $2.961(0.223)$ & 13.277 & $0.000 * * *$ & 0.314 \\
\hline & POS_HvL & 2 & 58 & $0.542(0.191)$ & 2.841 & 0.067 & 0.089 \\
\hline
\end{tabular}

${ }^{*} p<0.05 ;{ }^{*} p<0.01 ;{ }^{* *} p<0.001$. Given the significant effect of culture obtained in the between-subjects MANOVA reported in Table A7, indicating the presence

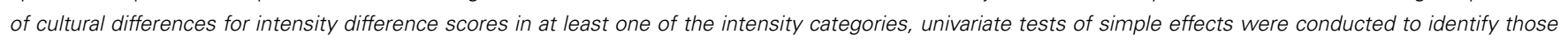

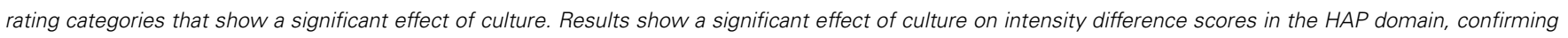
those reported in the main paper, as shown in bold.

Table A9 | Post hoc comparisons of intensity difference scores from ipsatized data.

\begin{tabular}{|c|c|c|c|c|c|}
\hline Dependent variable & (I) Culture & (J) Culture & Mean difference (I-J) & SE & $p$-Value \\
\hline \multirow[t]{4}{*}{ HAP H-L } & $J P$ & $\mathrm{RU}$ & -0.2627 & 0.15169 & 0.089 \\
\hline & & US & $-0.7526^{*}$ & 0.15009 & $0.000 * * *$ \\
\hline & & US & $-0.4899^{*}$ & 0.14408 & $0.001 * * *$ \\
\hline & US & JP & $0.7526^{*}$ & 0.15009 & $0.000 * * *$ \\
\hline \multirow{5}{*}{ POS H-L } & & US & -0.0982 & 0.13880 & 0.482 \\
\hline & $\mathrm{RU}$ & $J P$ & -0.2139 & 0.14028 & 0.133 \\
\hline & & US & $-0.3121^{*}$ & 0.13324 & $0.023^{*}$ \\
\hline & US & $J P$ & 0.0982 & 0.13880 & 0.482 \\
\hline & & RU & $0.3121^{*}$ & 0.13324 & $0.023^{*}$ \\
\hline \multirow{2}{*}{ LAP H-L } & US & $J P$ & -0.1704 & 0.16469 & 0.305 \\
\hline & & $R U$ & 0.1990 & 0.15809 & 0.213 \\
\hline
\end{tabular}

${ }^{*} p<0.05 ;{ }^{* *} p<0.01 ;{ }^{* * *} p<0.001$. Post hoc tests were performed to identify the effects of culture on intensity difference scores within each intensity category that showed significant or marginally non-significant effects. All results confirm those reported in the main paper as shown in bold. One additional marginally non-significant effect was observed, indicating that in the HAP domain, intensity difference scores between high and LA facial expressions of Japanese participants also differed from those of Russian participants. 


\section{ANALYSES OF CATEGORIZATION DIFFERENCES SCORES BASED ON IPSATIZED DATA}

Table A10 | Omnibus MANOVA for categorization difference scores of ipsatized data.

\begin{tabular}{|c|c|c|c|c|c|c|}
\hline Effect & Wilk's lambda & Hypothesis df & Error df & $\boldsymbol{F}$ & $p$-Value & $\eta_{p}^{2}$ \\
\hline Arousal_level $\times$ culture & 0.767 & 2.000 & 58.000 & $8.817^{a}$ & $0.000 * * *$ & 0.233 \\
\hline Intensity_category $\times$ culture & 0.793 & 4.000 & 114.000 & $3.497^{a}$ & $0.010 * *$ & 0.109 \\
\hline Arousal_level $\times$ intensity_category & 0.264 & 2.000 & 57.000 & $79.362^{a}$ & $0.000 * * *$ & 0.736 \\
\hline
\end{tabular}

${ }^{a}$ Exact statistic. ${ }^{*} p<0.05 ;{ }^{*} p<0.01 ;{ }^{* *} p<0.001$. Categorization difference score lobtained by subtracting one intensity category from another within the same arousal level, see main paper) were entered into a mixed three-way MANOVA with category difference (HAP-LAP, HAP-POS, POS-LAP) and arousal level (high, low) as within-subjects factors and culture (American, Japanese, Russian) as between-subjects factor. Results confirm those reported in the main paper: significant main effects were obtained for category difference and arousal level. There was also a near-significant main effect of culture $\left[F(2,58)=2.918, p=0.062, \eta^{2}=0.091\right]$. Culture interacted with the factors arousal level and category difference. A further two-way interaction was obtained between arousal level and category difference. Importantly, there was a significant three-way interaction between culture, category difference, and arousal level.

Table A11 | Between-subjects MANOVA to investigate the effects of culture on categorization difference scores from ipsatized data.

\begin{tabular}{lllllll}
\hline Effect & Wilk's lambda & $\boldsymbol{F}$ & Hypothesis $\mathbf{d f}$ & Error df & Sig. & $\boldsymbol{\eta}_{\boldsymbol{p}}^{\mathbf{2}}$ \\
\hline Culture & 0.539 & $4.981^{\mathrm{a}}$ & 8.000 & 110.000 & $0.000^{* * *}$ & 0.266 \\
\hline
\end{tabular}

${ }^{a}$ Exact statistic. ${ }^{*} p<0.05 ;{ }^{*} p<0.01 ;{ }^{* *} p<0.001$. Investigations of the nature of the three-way interaction obtained in the omnibus MANOVA focused on identifying the effects of culture on categorization difference scores based on ipsatized data and followed the following sequence: first, to investigate general effects of culture on intensity difference scores within each intensity category, a between-subjects MANOVA was performed with culture as predictor variable and category difference scores across arousal levelas multiple dependent variables Irating differences between HAP and LAP ratings for LA facial expressions: HAP-LAP_LA, HAP-POS_LA, POS-LAP_LA, HAP-LAP_HA, HAP_POS_HA, HAP-LAP_HAJ. Results confirm those reported in the main paper.

Table A12 | Univariate tests of simple effects of categorization difference scores from ipsatized data.

\begin{tabular}{|c|c|c|c|c|c|c|c|}
\hline Source & Dependent variable & Hypothesis df & Error df & Mean square (error) & $\boldsymbol{F}$ & $p$-Value & $\eta_{p}^{2}$ \\
\hline \multirow[t]{4}{*}{ Corrected model, culture } & L_HAPvLAP & 2 & 58 & $1.473(0.321)$ & 4.584 & $0.014^{*}$ & 0.136 \\
\hline & L_POSvLAP & 2 & 58 & $0.003(0.154)$ & 0.020 & 0.981 & 0.001 \\
\hline & H_HAPvLAP & 2 & 58 & $1.666(0.259)$ & 6.437 & $0.003^{* *}$ & 0.182 \\
\hline & H_POSvLAP & 2 & 58 & $0.381(0.176)$ & 2.161 & 0.124 & 0.069 \\
\hline
\end{tabular}

${ }^{*} p<0.05 ;{ }^{*} p<0.01 ;{ }^{* *} p<0.001$. Given the significant effect of culture obtained in the between-subjects MANOVA reported in Table A11, indicating the presence of cultural differences for at least on categorization difference score, univariate tests of simple effects were conducted to identify those rating category differences that show a significant effect of culture. Results confirm those reported in the main paper as shown in bold. 
Table A13 | Post hoc comparisons of categorization difference scores from ipsatized data.

\begin{tabular}{|c|c|c|c|c|c|}
\hline Dependent variable & (I) Culture & (J) Culture & Difference (I-J) & SE & $p$-Value \\
\hline \multirow[t]{5}{*}{ L_HAPvLAP } & $J P$ & $R U$ & 0.0666 & 0.18211 & 0.716 \\
\hline & US & & $0.4902^{*}$ & 0.18019 & $0.009 * *$ \\
\hline & US & & $0.4237^{*}$ & 0.17297 & $0.017^{*}$ \\
\hline & us & JP & $-0.4902^{*}$ & 0.18019 & $0.009 * *$ \\
\hline & RU & & $-0.4237^{*}$ & 0.17297 & $0.017 *$ \\
\hline \multirow{5}{*}{ L_HAPvPOS } & US & & $0.4987^{*}$ & 0.13508 & $0.000 * * *$ \\
\hline & $\mathrm{RU}$ & $J P$ & -0.0516 & 0.13652 & 0.707 \\
\hline & US & & $0.4471^{*}$ & 0.12967 & $0.001 * * *$ \\
\hline & US & JP & $-0.4987^{*}$ & 0.13508 & $0.000 * * *$ \\
\hline & RU & & $-0.4471^{*}$ & 0.12967 & $0.001 * * *$ \\
\hline \multirow{2}{*}{ H_HAPvLAP } & US & $J P$ & $0.4328^{*}$ & 0.16168 & $0.010 * *$ \\
\hline & RU & & -0.1328 & 0.15520 & 0.396 \\
\hline \multirow[t]{6}{*}{ H_POSVLAP } & $J P$ & $\mathrm{RU}$ & -0.1405 & 0.13486 & 0.302 \\
\hline & US & & $-0.2770^{*}$ & 0.13344 & $0.042^{*}$ \\
\hline & RU & $J P$ & 0.1405 & 0.13486 & 0.302 \\
\hline & US & & -0.1365 & 0.12809 & 0.291 \\
\hline & US & $J P$ & $0.2770^{*}$ & 0.13344 & $0.042^{*}$ \\
\hline & $R U$ & & 0.1365 & 0.12809 & 0.291 \\
\hline \multirow[t]{3}{*}{ H_HAPvPOS } & $J P$ & $\mathrm{RU}$ & $-0.4251^{*}$ & 0.10505 & $0.000 * * *$ \\
\hline & US & & -0.1558 & 0.10394 & 0.139 \\
\hline & RU & JP & $0.4251^{*}$ & 0.10505 & $0.000 * * *$ \\
\hline
\end{tabular}

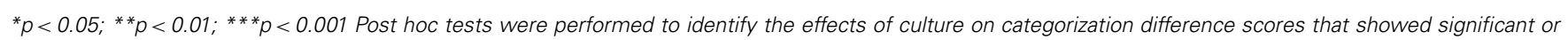
marginally non-significant effects. All results confirm those reported in the main paper as shown in bold. One additional marginally non-significant effect was observed,

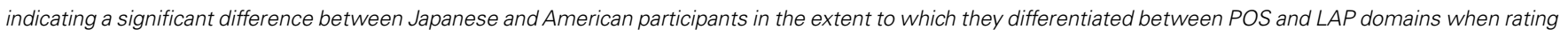
HA facial expressions. 


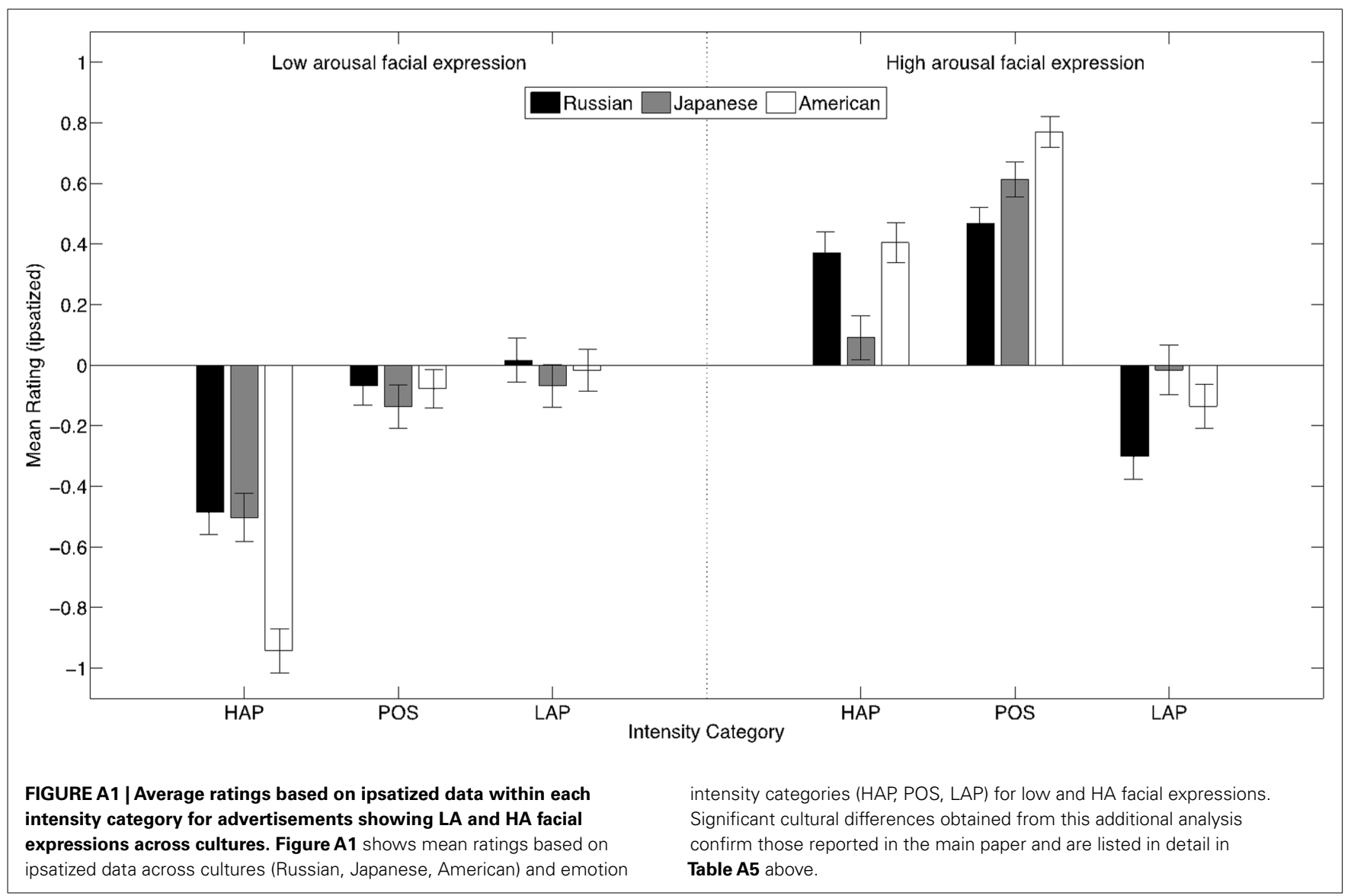




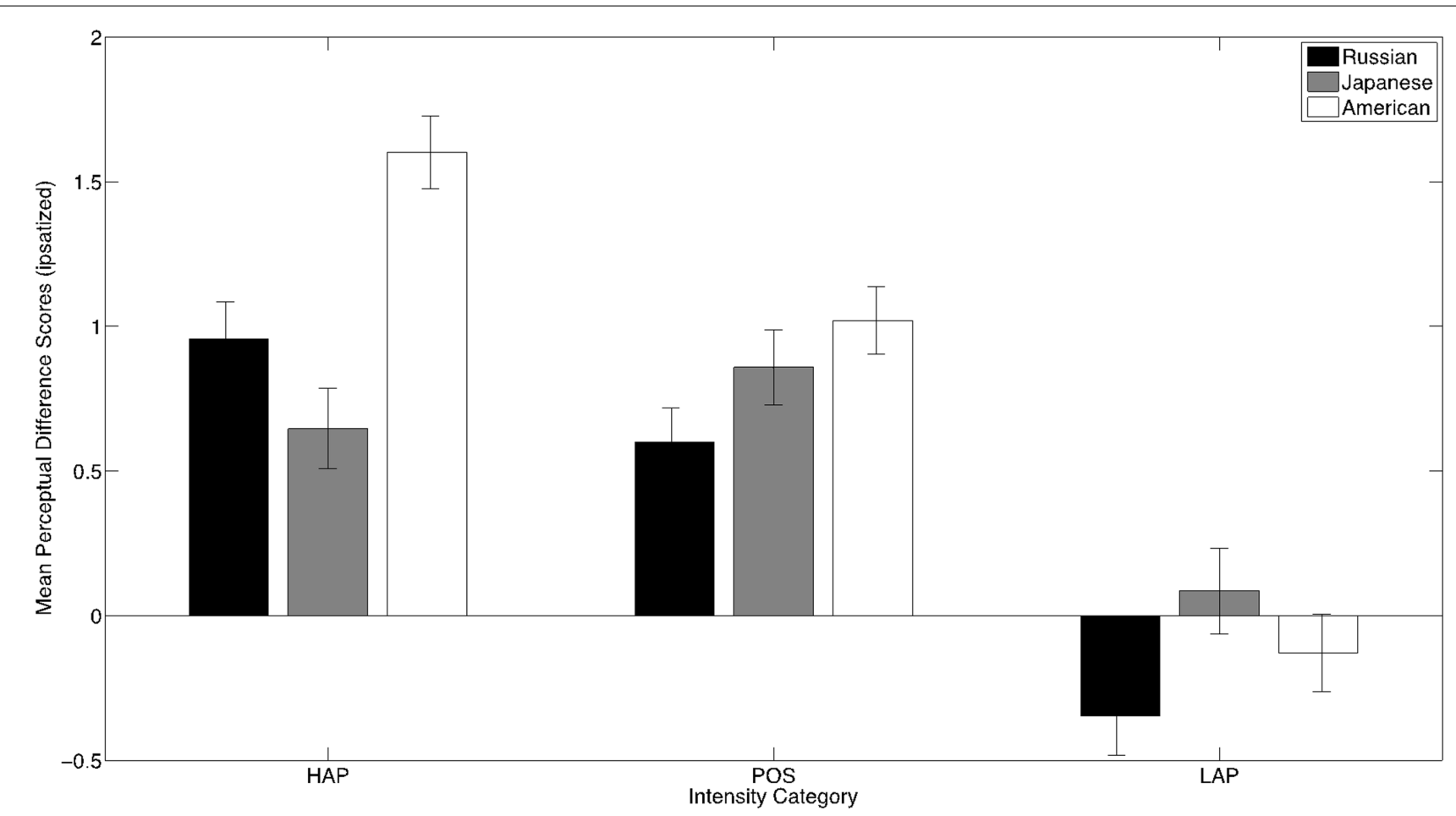

FIGURE A2 | Perceptual difference scores representing mean differences between ipsatized ratings for high compared to LA facial expressions in each intensity category across cultures. Significant cultural differences obtained from this additional analysis confirm those reported in the main paper and are listed in detail in Table $\mathbf{A 9}$ above.

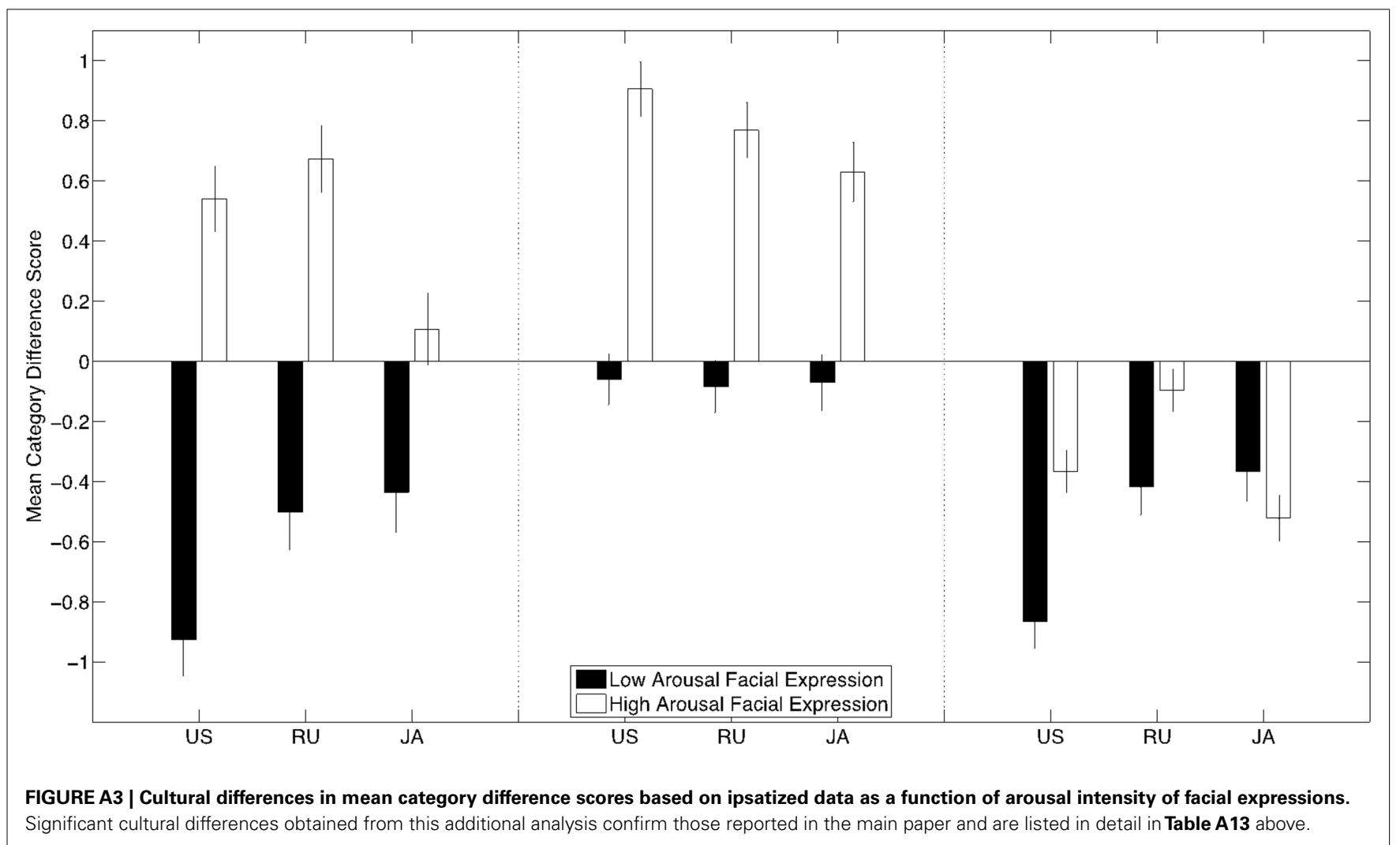

